\title{
TRUNCATED DERIVED FUNCTORS AND SPECTRAL SEQUENCES
}

\author{
HANS-JOACHIM BAUES, DAVID BLANC, AND BORIS CHORNY
}

\begin{abstract}
The $E_{2}$-term of the Adams spectral sequence may be identified with certain derived functors, and this also holds for a number of other spectral sequences. Our goal is to show how the higher terms of such spectral sequences are determined by truncations of relative derived functors, defined in terms of certain simplicial functors called mapping algebras.
\end{abstract}

\section{INTRODUCTION}

The various types of Adams spectral sequences, which play a central role in algebraic topology (cf. $\mathrm{A}, \mathrm{BCM}, \overline{\mathrm{BC}}, \overline{\mathrm{BK} 1}, \overline{\mathrm{BK} 2}, \mathrm{~N}, \mathrm{R}$, have a number of features in common:

(i) They are obtained from a space $\mathbf{Y}$ by constructing a (cosimplicial) resolution $\mathbf{Y} \rightarrow \mathbf{W}^{\bullet} \quad$ with respect to a spectrum $\mathcal{A}=\left\{A_{i}\right\}_{i=-\infty}^{\infty}$, with its associated cohomology theory $\mathcal{A}^{*}$.

(ii) The spectral sequence in question is the homotopy spectral sequence for $\mathbf{T W}^{\bullet}$, for a suitable homotopy functor $\mathbf{T}$.

(iii) The $E_{2}$-term of the spectral sequence can be identified as the derived functors of an algebraic functor $T$ associated to $\mathbf{T}$, applied to $\mathcal{A}^{*} \mathbf{Y}$.

The goal of this paper is to provide a description similar to (iii) for the $E_{n+2}$-term of the spectral sequence (for $n \geq 0$ ), as relative derived functors applied to the truncation $P^{n} \mathfrak{M}^{\mathcal{A}} \mathbf{Y}$ of a certain structure, called a mapping algebra, associated to $\mathbf{Y}$ (which reduces to $\mathcal{A}^{*} \mathbf{Y}$ when $n=0$ ).

Just as for the $E_{2}$-term, this has two advantages:

(a) The truncated mapping algebra $P^{n} \mathfrak{M}^{\mathcal{A}} \mathbf{Y}$ has less information than $\mathbf{Y}$ itself, but still enough to determine the $E_{n+2}$-term.

(b) Relative derived functors may be calculated using any resolution of $P^{n} \mathfrak{M}^{\mathcal{A}} \mathbf{Y}$.

The first author carried out this program for the $E_{3}$-term of the stable Adams spectral sequence in $\mathrm{Bau}, \mathrm{BJ} 2$, showing that extended calculations may be made using such a construction. See $\mathrm{BB}$ 3, $\mathrm{CF}$ for other general descriptions of the higher terms in the stable Adams spectral sequence, although not quite in the form of truncated derived functors as defined here.

Date: December 31, 2020.

2010 Mathematics Subject Classification. Primary: 55T99; secondary: 55U35, 18G50, 18C30.

Key words and phrases. Spectral sequence, relative derived functors, truncation, differentials, (co)simplicial resolutions, mapping algebra. 
0.1. Mapping algebras and truncations. By (iii) above, the $E_{2}$-term of the Adams spectral sequence depends only on the sets $\left[\mathbf{Y}, A_{i}\right]_{i \in \mathbb{Z}}$ and operations on them induced by homotopy classes of maps between (products of) the spaces $A_{i}$. This suggests that for the higher terms, we should look at the function $\operatorname{spaces}^{\operatorname{map}_{*}}\left(\mathbf{Y}, A_{i}\right)$, with additional structure induced by maps between the representing spaces. This structure is encoded by the notion of a mapping algebra: that is, a simplicial functor $\mathfrak{X}: \Theta^{\mathcal{A}} \rightarrow \mathcal{S}_{*}$ from the sub-simplicial category $\Theta^{\mathcal{A}}$ of Top $_{0}$ whose objects are products of copies of the various spaces $A_{i}$. For example, the realizable mapping algebra $\mathfrak{X}:=\mathfrak{M}^{\mathcal{A}} \mathbf{Y}$ has the value $\operatorname{map}(\mathbf{Y}, \mathbf{A})$ at each $\mathbf{A} \in \mathbf{\Theta}^{\mathcal{A}}$.

Mapping algebras admit truncations, defined by applying the Postnikov section functor $P^{n}$ to each mapping space. In particular, the 0-truncation contains the same information as the sets $\left[\mathbf{Y}, A_{i}\right]_{i \in \mathbb{Z}}$ of homotopy classes of maps, together with the operations on them induced by homotopy classes of maps between the spaces $A_{i}$ : this is precisely what was needed to determine the $E^{2}$-term as suitable derived functors in (iii) above.

This suggests that higher truncations of the mapping algebras may suffice to determine higher terms in the spectral sequence - depending, of course, on the homotopy functor $\mathbf{T}$ in question.

We may therefore summarize our program as follows:

(1) We need to show how a continuous functor $\mathbf{T}: \mathrm{Top}_{*} \rightarrow \mathrm{Top}_{*}$ factors through the category $\mathrm{Map}^{A}$ of mapping algebras as $\mathfrak{T}^{*} \mathfrak{M}^{\mathcal{A}}$, for a suitable homotopy functor $\mathfrak{T}: \mathrm{Map}^{A} \rightarrow \mathrm{Top}_{*}$.

(2) We want $\mathfrak{W}_{\bullet}:=\mathfrak{M}^{\mathcal{A}} \mathbf{W}^{\bullet}$ to be a resolution of $\mathfrak{M}^{\mathcal{A}} \mathbf{Y}$ in the resolution model category of simplicial mapping algebras, in order to guarantee that both the (functorial) cosimplicial resolution $\mathbf{W}^{\bullet}$ of $\mathbf{Y}$, and the resulting cosimplicial space $\mathbf{T W}^{\bullet}$, are homotopy functors of $\mathfrak{M}^{\mathcal{A}} \mathbf{Y}$. This will let us identify $\mathbf{T W}^{\bullet}$ as a certain relative left derived functor $\left(\mathbf{L}^{\text {rel }} \mathfrak{T}\right) \mathfrak{M}^{\mathcal{A}} \mathbf{Y}=\mathfrak{T} \mathfrak{W}$. of $\mathfrak{T}$ applied to the mapping algebra $\mathfrak{M}^{\mathcal{A}} \mathbf{Y}$ (see 33.1 ).

(3) Finally, we must show that in the cases of interest to us, the $E_{r}$-term of the homotopy spectral sequence for $\mathbf{T W}^{\bullet}=(\mathbf{L} \mathfrak{T}) \mathfrak{M}^{\mathcal{A}} \mathbf{Y}$ depends only on the $n$-truncation $P^{r+2} \mathfrak{W}_{\bullet}$, for each $r \geq 2$. Functors $\mathbf{T}$ with this property are called level.

0.2. Remark. There are also a number of less familiar spectral sequences obtained dually by constructing a simplicial resolution $\mathbf{X} \bullet \rightarrow \mathbf{Y}$ with respect to $\mathcal{B}=\left\{\mathbf{S}^{i}\right\}_{i=1}^{\infty}$, applying a homotopy functor $\mathbf{T}: \mathcal{C} \rightarrow \mathcal{C}$, and then using the homotopy spectral sequence for the simplicial space TX. (see [Sto, Bl1, DKSS]). Here too, one can identify the $E^{2}$-term with the derived functors of an algebraic functor of $\pi_{*} \mathbf{Y}$ (the algebraic object corepresented by $\mathcal{B}$ ). We include these in the paper mainly in order to show that the formalism we describe here is not limited to the Adams spectral sequence, even though this is our most important example. Moreover, in a number of ways the simplicial-covariant version is cleaner than the cosimplicial-contravariant one.

However, since Eckmann-Hilton duality is not formal, we are forced to work carefully through the details in the two versions separately: for this reason, each section is divided into two parts, starting with the covariant case. 
For reasons of space, we deal here only with the unstable spectral sequences. For the stable analogue, we must choose a simplicial model category of spectra (cf. BF, EKMM, HSS, L) and work there throughout; one can still take Postnikov $n$-sections of the mapping spaces $\operatorname{map}_{*}\left(\mathcal{B}, \mathbf{X}_{\bullet}\right)$.

0.3. Outline. In Section 1 we define enriched sketches and the associated mapping algebras (as well as the dual versions). It turns out that we have competing versions of mapping algebras: the category $\mathrm{sMap}_{\mathrm{re}}^{\mathrm{St}, R}$, which allows us to factor $\mathbf{T}$ as $\mathfrak{T} \circ$ $\mathfrak{M}^{\mathcal{A}}$ in $\oint 0.1(1)$, is not right proper, so we need a variant $\mathcal{S}_{*}^{\Theta^{\mathcal{A}}}$ in which $\mathfrak{W}_{\bullet}:=$ $\mathfrak{M}^{\mathcal{A}} \mathbf{W}^{\bullet}$ is indeed a cofibrant replacement for $\mathfrak{M}^{\mathcal{A}} \mathbf{Y}$ in the resolution model category $\mathcal{S}_{*}^{\Theta^{\mathcal{A}} \times \Delta^{\mathrm{op}}}$.

In Section 2 we construct the category $\mathrm{sMap}_{\mathrm{re}}^{\mathrm{St}, R}$ of mapping algebras, for $\mathcal{A}$ the Eilenberg-Mac Lane spectrum for a commutative ring $R$, and show:

Theorem A. There is a realization functor $N:\left(\mathrm{sMap}_{\mathrm{re}}^{\mathrm{St}, R}\right)^{\mathrm{op}} \rightarrow \mathcal{S}_{*}$, equipped with a natural weak equivalence $\mathfrak{M}^{\mathcal{A}} \circ N \rightarrow$ Id.

See Theorem 2.22 and Corollary 2.23 below.

Thus any homotopy functor $\mathbf{T}: \mathrm{Top}_{*} \rightarrow \mathrm{Top}_{*} \quad$ which preserves $R$-equivalences, when restricted to $R$-good spaces, induces a functor $\mathfrak{T}:=\mathbf{T} \circ N:\left(\operatorname{sMap}_{\mathrm{re}}^{\mathrm{St}, R}\right)^{\mathrm{op}} \rightarrow$ Top $_{*}$ equipped with a natural weak equivalence $\mathfrak{T} \circ \mathfrak{M}^{\mathrm{St}, R} \rightarrow \mathbf{T}$.

When $\mathcal{B}$ is the sphere spectrum (cf. 80.2 ), there is a dual category $\operatorname{sMap}_{\mathrm{St}}$ of $\mathcal{B}$-mapping algebras with a realization functor $N: \mathrm{sMap}_{\mathrm{St}} \rightarrow \mathrm{Top}_{*}$, (see Theorem 2.13 and Corollary 2.14).

In Section 3 we define the general notion of a relative derived functor (\$3.1), and show how it applies to the functor $\mathfrak{T}:\left(\mathrm{sMap}_{\mathrm{re}}^{\mathrm{St}, R}\right)^{\mathrm{op}} \rightarrow \mathrm{Top}_{*}$ associated to the homotopy functor $\mathbf{T}: \mathrm{Top}_{*} \rightarrow \mathrm{Top}_{*}$. To do so, we have to relate the two types of mapping algebras described in Section 1 - those that are used for resolutions, and those for which $\mathfrak{T}$ is defined - by means of Theorem 3.21, which implies:

Theorem B. If $\mathbf{Y}$ is $R$-good, any simplicial resolution $\mathfrak{V}_{\bullet}$ of $\mathfrak{M}^{\mathrm{St}, R} \mathbf{Y}$ in the resolution model category $\mathcal{S}_{*}^{\boldsymbol{\Theta}^{\mathcal{A}} \times \Delta^{\mathrm{oP}}}$ is Reedy weakly equivalent (i.e., in each simplicial dimension) to a simplicial object $\mathfrak{W}_{\bullet}$ in $\left(\mathrm{sMap}_{\mathrm{re}}^{\mathrm{St}, R}\right)^{\Delta^{\mathrm{op}}}$.

The dual version, for the sphere spectrum, is Theorem 3.10

Finally, in Section 4 we deal with the truncated versions of our higher derived functors, explain what data is needed to determine the $E_{r}$-term of the homotopy spectral sequence of a (co)simplicial space by formalizing the notion of a level functor $(\$ 4.2)$, and show

Theorem C. For $R=\mathbb{F}_{p}$ or $\mathbb{Q}, \quad \mathbf{Z} \in \mathcal{S}_{*}$, and $R$-good $\mathbf{Y}$, the unstable Adams spectral sequence for $\operatorname{map}_{*}(\mathbf{Z}, \mathbf{Y})$ is determined by a simplicial mapping algebra resolution $\mathfrak{W}_{\bullet}$ of $\mathfrak{M}^{\mathrm{St}, R} \mathbf{Y}$, and for each $r \geq 2$ the $E_{r}$-term is determined by the corresponding $(r-2)$-truncated mapping algebras.

See Theorem 4.13

This implies that the mapping space functor $\operatorname{map}_{*}(\mathbf{Z},-)$ is a level homotopy functor on $R$-good spaces. We also prove a number of similar results for functors related to the sphere spectrum (see Propositions 4.8, 4.9, and 4.10). 
0.4. Notation. The category of finite ordered sets and order-preserving maps will be denoted by $\Delta$ (cf. [Ma2, §2]), so a simplicial object $G_{\bullet}$ in $\mathcal{C}$ is a functor $\Delta^{\mathrm{op}} \rightarrow \mathcal{C}$, and the category of such will be denoted by $\mathcal{C}^{{ }^{\mathrm{op}}}$. Similarly, a cosimplicial object $G^{\bullet}$ in a category $\mathcal{C}$ is a functor $\Delta \rightarrow \mathcal{C}$, and the category of such will be denoted by $\mathcal{C}^{\Delta}$. There is a natural embedding $c(-) \cdot \mathfrak{C} \rightarrow \mathcal{C}^{\Delta^{\mathrm{op}}}$ (the constant simplicial object), and similarly $c(-)^{\bullet}: \mathcal{C} \rightarrow \mathcal{C}^{\Delta}$.

Write $\Delta_{+}$for the subcategory of $\Delta$ with the same objects but only monic maps. A functor $G: \Delta_{+}^{\mathrm{op}} \rightarrow \mathcal{C}$ (respectively, $G: \Delta_{+} \rightarrow \mathcal{C}$ ) is called a restricted (co)simplicial object in $\mathcal{C}$. The inclusion $i: \Delta_{+} \hookrightarrow \Delta$ induces a forgetful functor $i^{*}: \mathcal{C}^{\Delta^{\text {op }}} \rightarrow \mathcal{C}^{\Delta_{+}^{\text {op }}}$, which has a left adjoint $\mathcal{L}: \mathcal{C}^{\Delta_{+}^{\text {op }}} \rightarrow \mathcal{C}^{\Delta^{\text {op }}} \quad$ (for suitable $\mathcal{C}$ ).

The category of topological spaces will be denoted by Top, that of pointed spaces by $\mathrm{Top}_{*}$, and that of pointed connected spaces by $\mathrm{Top}_{0}$. The category of simplicial sets will be denoted by $\mathcal{S}=\operatorname{Set}^{\Delta^{\mathrm{op}}}$, that of pointed simplicial sets by $\mathcal{S}_{*}=\operatorname{Set}_{*}^{\Delta^{\mathrm{op}}}$, that of simplicial groups by $\mathcal{G}=\mathrm{Gp}^{\Delta^{\mathrm{op}}}$. Write $\operatorname{map}_{*}(\mathbf{X}, \mathbf{Y})$ for the standard function complex in $\mathcal{S}_{*}, \mathrm{Top}_{0}$, or $\mathcal{G}$ (see [GJ] I, §1.5]). Note that both $\mathrm{Top}_{0}$ and $\mathcal{S}_{*}$ are enriched over $\left(\mathcal{S}_{*}, \wedge\right)$, but if we forget the basepoints, the same mapping spaces $\operatorname{map}_{\mathcal{S}_{*}}(X, Y)$ or map Top $(X, Y)$ also define an enrichment over $(\mathcal{S}, \times)$, which is the one we shall use (see $[\underline{\mathrm{H}}, 9.1 .14]$ ),

We denote the category of pointed Kan complexes by $\mathcal{S}^{\text {Kan }}$, that of reduced simplicial sets (with a single vertex) by $\mathcal{S}^{\text {red }}$, and the full subcategory of $n$-types in $\mathcal{S}_{*} \quad$ - i.e., spaces $X$ with $\pi_{i}(X, x)=0$ for $i>n$ and all $x \in X_{0} \quad$ - by $\mathcal{S}_{[n]}$, with $P^{n}: \mathcal{S}_{*} \rightarrow \mathcal{S}_{[n]}$ the $n$-th Postnikov section functor.

0.5. Acknowledgments. The research of the second and third authors was partially supported by Israel Science Foundation grants 770/16 and 1138/16, respectively.

\section{MAPPING ALGEBRAS}

The main technical tool in our approach is the notion of a mapping algebra, first introduced in [BB2, §8]. We shall need a number of variants of this notion, together with their dual versions.

\section{A. Enriched sketches and mapping algebras}

1.1. Definition. Let $\mathcal{C}$ be a pointed simplicial model category, $\mathcal{B}$ a set of fibrant and cofibrant homotopy cogroup objects in $\mathcal{C}, \mathcal{F}$ a category of finite simplicial sets, and $\mathcal{E}$ a set of cocones in $\mathcal{C}$. The associated enriched sketch, or multi-sorted theory (cf. Bor, §5.6]) $\boldsymbol{\Theta}_{\mathcal{B}}=\boldsymbol{\Theta}_{(\mathcal{B}, \mathcal{F}, \mathcal{E})}$ is the smallest full sub-simplicial category of $\mathcal{C}$ containing $\mathcal{B}$ and closed under the operations $-\otimes K$ for $K \in \mathcal{F}$ and taking colimits of the cocones in $\mathcal{E}$. In this setting:

(1) A $\mathcal{B}$-presheaf is a pointed simplicial functor $\mathfrak{X}: \Theta_{\mathcal{B}}^{\text {op }} \rightarrow \mathcal{S}_{*}$. The category of all $\mathcal{B}$-presheaves is denoted by $\mathcal{S}_{*}^{\boldsymbol{\Theta}_{\mathcal{B}}^{\text {op }}}$, and the value of $\mathfrak{X}$ at $\mathbf{B} \in \boldsymbol{\Theta}_{\mathcal{B}} \quad$ will be written $\mathfrak{X}\{\mathbf{B}\}$.

A map $\mathfrak{f}: \mathfrak{X} \rightarrow \mathfrak{Y}$ of $\mathcal{B}$-presheaves is called a weak equivalence if $\mathfrak{f}\{\mathbf{B}\}: \mathfrak{X}\{\mathbf{B}\} \rightarrow \mathfrak{Y}\{\mathbf{B}\} \quad$ is a weak equivalence for each $\mathbf{B} \in \mathbf{\Theta}_{\mathcal{B}}$. Two $\mathcal{B}$ presheaves are said to be weakly equivalent if they are connected by a finite zigzag of weak equivalences. 
(2) A strict $\mathcal{B}$-mapping algebra is a $\mathcal{B}$-presheaf $\mathfrak{X}$ for which the natural maps

$$
\mathfrak{X}\{\mathbf{B} \otimes K\} \rightarrow \mathfrak{X}\{\mathbf{B}\}^{K} \quad \text { and } \quad \mathfrak{X}\left\{\operatorname{colim}_{i \in I} \mathbf{B}_{i}\right\} \rightarrow \lim _{i \in I} \mathfrak{X}\left\{\mathbf{B}_{i}\right\}
$$

are isomorphisms for all $\mathbf{B} \in \mathbf{\Theta}_{\mathcal{B}}, \quad K \in \mathcal{F}$, and diagrams $I$ in $\mathcal{E}$. The full subcategory of strict $\mathcal{B}$-mapping algebras will be denoted by $\operatorname{sMap}_{\mathcal{B}}$.

(3) A weak $\mathcal{B}$-mapping algebra is a $\mathcal{B}$-presheaf $\mathfrak{X}$ which is weakly equivalent to a strict $\mathcal{B}$-mapping algebra. Thus in particular, the maps of (1.2) are weak equivalences. The full subcategory of weak $\mathcal{B}$-mapping algebras will be denoted by wMap $_{\mathcal{B}}$.

1.3. Remark. In principle, we would like to identify a weak $\mathcal{B}$-mapping algebra more conceptually as a $\mathcal{B}$-presheaf for which not only the maps of (1.2) are weak equivalences, but also appropriate higher coherences hold. However, as we shall not in fact need to work explicitly with weak $\mathcal{B}$-mapping algebras, we can make do here with the above ad hoc definition.

1.4. Example. The main example of an enriched sketch we shall consider in this paper is the case where $\mathcal{C}=\operatorname{Top}_{0}, \quad \mathcal{B}=\left\{\mathbf{S}^{n}\right\}_{n=1}^{\infty}$ and $\mathcal{F}$ consists of the inclusions $i_{0}, i_{1}: \Delta[0] \hookrightarrow \Delta[1]$, The cocone collection $\mathcal{E}$ contains all coproducts of cardinality $<\lambda$ for some fixed limit cardinal $\lambda$ (e.g., $\aleph_{0}$ ), and the pushout squares
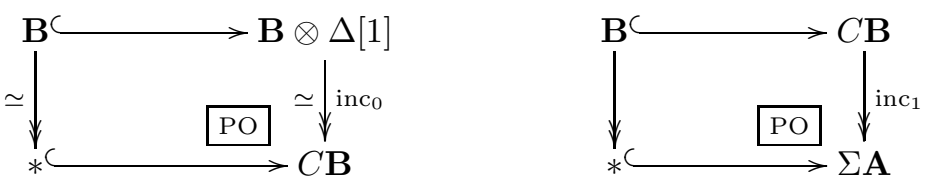

for $\mathbf{B} \in \boldsymbol{\Theta}_{\mathcal{B}}$. (These will be our models for the cone $C X$ and suspension $\Sigma X$ of any $X \in \mathcal{C})$.

Thus a strict $\mathcal{B}$-mapping algebra $\mathfrak{X}$ will take the two squares of (1.5) to pullback squares:
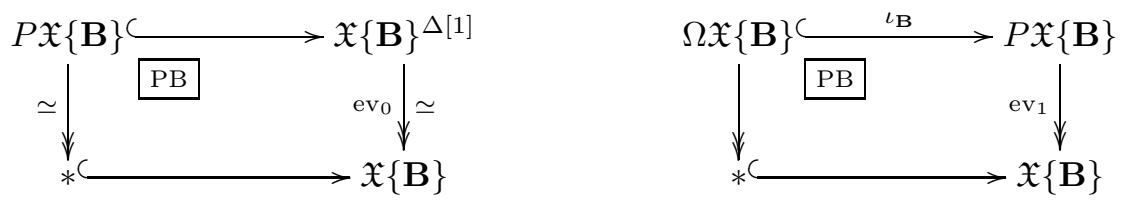

One might also consider localized versions, where $\mathcal{B}=\left\{\mathbf{S}_{R}^{n}\right\}_{n=1}^{\infty}$ for some subring $R \subseteq \mathbb{Q} \quad$ (cf. $[\mathrm{Bi})$. In particular, when $R=\mathbb{Q}$ we may replace $\mathcal{C}=$ Top $_{0}$ by a suitable algebraic model of rational homotopy types, such as the category of differential graded Lie algebras.

More generally, one could take any space $\mathbf{M} \in$ Top $_{0}$, and let $\mathcal{B}=\left\{\Sigma^{n} \mathbf{M}\right\}_{n=1}^{\infty}$. However, while the formal part of our program can be made to work in this case (see [BBD]), the application to the homotopy spectral sequence of a simplicial space is not available for $\mathbf{M}$ which is not essentially a sphere (see [CDI] and [B12, $\S 4.6]$ ).

1.7. Definition. For any enriched sketch $\boldsymbol{\Theta}_{\mathcal{B}}$ as above, the most important example of a $\mathcal{B}$-presheaf $\mathfrak{X}$ is a realizable one, associated to an object $\mathbf{Y} \in \mathcal{C}$, where $\mathfrak{X}\{\mathbf{B}\}:=$ $\operatorname{map}_{\mathcal{C}}(\mathbf{B}, \mathbf{Y})$ for any $\mathbf{B} \in \boldsymbol{\Theta}_{\mathcal{B}}$. Evidently, this will be a strict $\mathcal{B}$-mapping algebra, which we denote by $\mathfrak{M}_{\mathcal{B}} \mathbf{Y}$ (of course, it actually takes all colimits in $\boldsymbol{\Theta}_{\mathcal{B}}$ to the corresponding limits). When $\mathbf{Y} \in \operatorname{Obj} \boldsymbol{\Theta}_{\mathcal{B}}$, we say that $\mathfrak{M}_{\mathcal{B}} \mathbf{Y}$ is free. 
The strong Yoneda Lemma for enriched categories (see [K, 2.4]) implies:

1.8. Lemma. If $\mathfrak{Y}$ is a $\mathcal{B}$-presheaf and $\mathfrak{M}_{\mathcal{B}} \mathbf{B}$ is a free strict $\mathcal{B}$-mapping algebra (for $\mathbf{B} \in \mathbf{\Theta}_{\mathcal{B}}$ ), there is a natural isomorphism

$$
\Phi: \operatorname{map}_{\mathcal{S}_{*}^{\Theta_{\mathcal{B}}}}{ }_{\Theta^{\text {op }}}\left(\mathfrak{M}_{\mathcal{B}} \mathbf{B}, \mathfrak{Y}\right) \stackrel{\cong}{\rightrightarrows} \mathfrak{Y}\{\mathbf{B}\},
$$

with $\Phi(\mathfrak{f})=\mathfrak{f}\left(\operatorname{Id}_{\mathbf{B}}\right) \in \mathfrak{Y}\{\mathbf{B}\}_{0}$ for any $\mathfrak{f} \in \operatorname{Hom}_{\mathcal{S}_{*}^{\Theta_{\mathcal{B}}^{\text {op }}}}\left(\mathfrak{M}_{\mathcal{B}} \mathbf{B}, \mathfrak{Y}\right)=\operatorname{map}_{\mathcal{S}_{*} \Theta_{\mathcal{B}}^{\text {op }}}\left(\mathfrak{M}_{\mathcal{B}} \mathbf{B}, \mathfrak{Y}\right)_{0}$.

1.9. Remark. It is sometimes convenient think of a $\mathcal{B}$-presheaf $\mathfrak{X}$ as a category $\mathcal{X}$ with object set $\mathcal{O}:=\operatorname{Obj}\left(\Theta_{\mathcal{B}}\right) \cup\{\star\}$, enriched in pointed simplicial sets as follows:

$$
\operatorname{map}_{\mathcal{X}}(\mathbf{A}, \mathbf{B})= \begin{cases}\operatorname{map}_{\boldsymbol{\Theta}_{\mathcal{B}}}(\mathbf{A}, \mathbf{B}) & \text { if } \mathbf{A}, \mathbf{B} \in \operatorname{Obj}\left(\mathbf{\Theta}_{\mathcal{B}}\right) \\ \mathfrak{X}\{\mathbf{A}\} & \text { if } \mathbf{A} \in \operatorname{Obj}\left(\mathbf{\Theta}_{\mathcal{B}}\right) \text { and } \mathbf{B}=\star \\ c\left(\left\{*, \mathrm{Id}_{\star}\right\}\right) \bullet & \text { if } \mathbf{A}=\mathbf{B}=\star \\ c(\{*\}) \bullet & \text { otherwise. }\end{cases}
$$

Thus a realizable $\mathcal{B}$-presheaf $\mathfrak{X}=\mathfrak{M}_{\mathcal{B}} \mathbf{Y}$ corresponds to a sub-simplicial category $\mathcal{X}$ of $\mathcal{C}$ with object set $\operatorname{Obj}\left(\boldsymbol{\Theta}_{\mathcal{B}}\right) \cup\{\mathbf{Y}\} \quad$ (compare [BB2, §8.1]).

1.11. Definition. An enriched sketch $\Theta_{\mathcal{B}}$ in a model category $\mathcal{C}$ has an algebraic version, which is the (ordinary) sketch $\Theta_{\mathcal{B}}:=\pi_{0} \boldsymbol{\Theta}_{\mathcal{B}}$ - that is, $\Theta_{\mathcal{B}}$ has the same objects as $\boldsymbol{\Theta}_{\mathcal{B}}$, and $\operatorname{Hom}_{\Theta_{\mathcal{B}}}\left(\mathbf{B}, \mathbf{B}^{\prime}\right):=\pi_{0} \operatorname{map}_{\boldsymbol{\Theta}_{\mathcal{B}}}\left(\mathbf{B}, \mathbf{B}^{\prime}\right)$. An algebra (or model) for $\Theta_{\mathcal{B}}$ is a functor $\Lambda: \Theta_{\mathcal{B}}^{\text {op }} \rightarrow$ Set which takes the coproduct of any discrete cocone in $\mathcal{E}$ to a product in Set (see [Bor, §5.6]).

These are called $\Pi_{\mathcal{B}}$-algebras, and the category of such is denoted by $\Pi_{\mathcal{B}}-\mathrm{Alg}$ : for $\mathcal{B}=\left\{\mathbf{S}^{n}\right\}_{n=1}^{\infty}$, these are simply the $\Pi$-algebras of DK2]. Note that if $\mathfrak{X}$ is a (weak or strict) $\mathcal{B}$-mapping algebra, then $\pi_{0} \mathfrak{X}$ is a $\Pi_{\mathcal{B}}$-algebra; the same need not hold for an arbitrary $\mathcal{B}$-presheaf. We say that a $\Pi_{\mathcal{B}}$-algebra $\Lambda$ is realizable if it is of the form $\pi_{0} \mathfrak{M}_{\mathcal{B}} \mathbf{Y}$ for some $\mathbf{Y} \in \mathcal{C}$. A coproduct of $\Pi_{\mathcal{B}}$-algebras of the form $\pi_{0} \mathfrak{M}_{\mathcal{B}} \mathbf{B}$ for $\mathbf{B} \in \operatorname{Obj} \boldsymbol{\Theta}_{\mathcal{B}}$ is called free.

\section{B. Dual sketches and mapping algebras}

There are dual versions of all three notions, defined as follows:

1.12. Definition. Let $\mathcal{C}$ be a pointed simplicial model category, $\mathcal{A}$ a set of fibrant and cofibrant homotopy group objects in $\mathcal{C}, \mathcal{F}$ a category of finite simplicial sets, and $\mathcal{L}$ a set of cones in $\mathcal{C}$. The associated dual enriched sketch $\boldsymbol{\Theta}^{\mathcal{A}}=\boldsymbol{\Theta}^{(\mathcal{A}, \mathcal{K}, \mathcal{L})}$ is the smallest full sub-simplicial category of $\mathcal{C}$ containing $\mathcal{A}$ and closed under the operations $(-)^{K}$ for $K \in \mathcal{F}$ and taking limits of the cones in $\mathcal{L}$. In this setting:

(1) An $\mathcal{A}$-dual presheaf is a pointed simplicial functor $\mathfrak{X}: \Theta^{\mathcal{A}} \rightarrow \mathcal{S}_{*}$. The category of $\mathcal{A}$-dual presheaves is denoted by $\mathcal{S}_{*}^{\Theta^{\mathcal{A}}}$, and the value of $\mathfrak{X}$ at $\mathbf{A} \in \mathbf{\Theta}^{\mathcal{A}}$ will again be written $\mathfrak{X}\{\mathbf{A}\}$.

(2) A dual strict $\mathcal{A}$-mapping algebra is a $\mathcal{A}$-dual presheaf $\mathfrak{X}$ for which the natural maps

$$
\mathfrak{X}\left\{\mathbf{A}^{K}\right\} \rightarrow \mathfrak{X}\{\mathbf{A}\}^{K} \quad \text { and } \quad \mathfrak{X}\left\{\lim _{i \in I} \mathbf{A}_{i}\right\} \rightarrow \lim _{i \in I} \mathfrak{X}\left\{\mathbf{A}_{i}\right\}
$$

are isomorphisms for all $\mathbf{A}, \mathbf{A}_{i} \in \mathbf{\Theta}^{\mathcal{A}}, \quad K \in \mathcal{F}$, and diagrams $I$ in $\mathcal{L}$. The subcategory of dual strict $\mathcal{A}$-mapping algebras will be denoted by sMap $^{A}$. 
(3) A dual weak $\mathcal{A}$-mapping algebra is a $\mathcal{A}$-dual presheaf $\mathfrak{X}$ which is weakly equivalent to a dual strict $\mathcal{A}$-mapping algebra, so in particular, the maps of (1.13) are weak equivalences (see Remark 1.3 above). The subcategory of dual weak $\mathcal{A}$-mapping algebras will be denoted by wMap $^{A}$.

1.14. Example. The main example of an enriched dual sketch we consider here is the $\Omega$-spectrum case, where $\mathcal{C}=\mathcal{S}_{*}$ and $\mathcal{A}=\left\{\mathbf{A}_{n}\right\}_{n=-\infty}^{\infty}$ are the spaces of an $\Omega$-spectrum $A$ (in the sense of $[\mathrm{BF}]$ ). The category $\mathcal{F}$ then consists of the inclusions $i_{0}, i_{1}: \Delta[0] \hookrightarrow \Delta[1]$, and the cone collection $\mathcal{L}$ contains all products of cardinality $<\lambda$ for some fixed limit cardinal $\lambda$ and the pullback squares
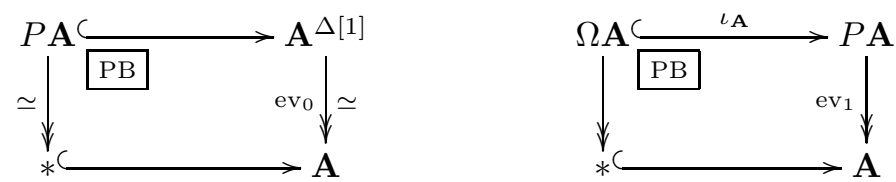

for any $\mathbf{A} \in \mathbf{\Theta}^{\mathcal{A}}$. Thus a dual strict $\mathcal{A}$-mapping algebra $\mathfrak{X}$ will take the two pullback squares of (1.15) to those of (1.6).

More generally, one might take any set of $\Omega$-spectra - in particular, the set of all $A$-module spectra of bounded cardinality, for a fixed ring spectrum $A$.

1.16. Definition. For any dual enriched sketch $\Theta^{\mathcal{A}}$, the realizable dual strict $\mathcal{A}$ mapping algebra $\mathfrak{X}$ associated to $\mathbf{Y} \in \mathcal{C}$ has $\mathfrak{X}\{\mathbf{A}\}:=\operatorname{map}_{\mathcal{C}}(\mathbf{Y}, \mathbf{A})$ for each $\mathbf{A} \in \boldsymbol{\Theta}_{\mathcal{B}}$. We will denote it by $\mathfrak{M}^{\mathcal{A}} \mathbf{Y}$. When $\mathbf{Y} \in \operatorname{Obj} \boldsymbol{\Theta}^{\mathcal{A}}$, we again say that $\mathfrak{M}^{\mathcal{A}} \mathbf{Y}$ is free.

The analogue of Lemma 1.8 also holds:

1.17. Lemma (cf. [BS2, Lemma 1.12]). If $\mathfrak{Y}$ is an $\mathcal{A}$-dual presheaf and $\mathfrak{M}^{\mathcal{A}} \mathbf{A}$ is a free dual strict $\mathcal{A}$-mapping algebra (for $\mathbf{A} \in \mathbf{\Theta}^{\mathcal{A}}$ ), there is a natural isomorphism

$$
\Phi: \operatorname{map}_{\mathcal{S}_{*} \Theta^{\mathcal{A}}}\left(\mathfrak{M}^{\mathcal{A}} \mathbf{A}, \mathfrak{Y}\right) \stackrel{\cong}{\longrightarrow} \mathfrak{Y}\{\mathbf{A}\}
$$

with $\Phi(\mathfrak{f})=\mathfrak{f}\left(\operatorname{Id}_{\mathbf{A}}\right) \in \mathfrak{Y}\{\mathbf{A}\}_{0} \quad$ for any $\mathfrak{f} \in \operatorname{Hom}_{\mathcal{S}_{*} \mathcal{A}}\left(\mathfrak{M}^{\mathcal{A}} \mathbf{A}, \mathfrak{Y}\right)$.

1.18. Definition. As in 11.11 , given a dual enriched sketch $\Theta^{\mathcal{A}}$, the corresponding "algebraic" sketch $\Theta^{\mathcal{A}}:=\pi_{0} \Theta^{\mathcal{A}}$, whose models are now functors $\Lambda: \Theta^{\mathcal{A}} \rightarrow$ Set preserving all products among the cones listed in $\mathcal{E}$. These will be called $\Pi^{\mathcal{A}}$-algebras, and their category will denoted by $\Pi^{\mathcal{A}}-\mathrm{Alg}$. Again, if $\mathfrak{X}$ is a (weak or strict) mapping algebra, then $\pi_{0} \mathfrak{X}$ is a $\Pi^{\mathcal{A}}$-algebra. A $\Pi^{\mathcal{A}}$-algebra is realizable if it is isomorphic to $\pi_{0} \mathfrak{M}^{\mathcal{A}} \mathbf{Y}$ for some $\mathbf{Y} \in \mathcal{C}$, and it is free if it is of the form $\pi_{0} \mathfrak{M}^{\mathcal{A}} \mathbf{A}$ for $\mathbf{A} \in \operatorname{Obj} \Theta^{\mathcal{A}}$.

1.19. Example. When $\mathcal{A}=\left\{K\left(\mathbb{F}_{p}, i\right)\right\}_{i=1}^{\infty} \quad$ and $\lambda=\aleph_{0}, \quad \boldsymbol{\Theta}^{\mathcal{A}} \quad$ is the simplicial category of finite type $\mathbb{F}_{p}$-GEMs, and a $\Pi^{\mathcal{A}}$-algebra is simply an unstable algebra over the $\bmod p$ Steenrod algebra (cf. $[\mathrm{Sc}]$ ).

1.20. Model categories of mapping algebras. Like all categories of simplicial functors with small indexing category, the (dual) presheaf categories $\mathcal{S}_{*}^{\boldsymbol{\Theta}_{\mathcal{B}}^{\text {op }}}$ and $\mathcal{S}_{*}^{\Theta^{\mathcal{A}}}$ have proper simplicial model category structures (see [H] 13.1.14]), in which the fibrations and weak equivalences are defined objectwise (see [DK1, §1]). Thus 
a map $\mathfrak{f}: \mathfrak{X} \rightarrow \mathfrak{Y}$ of $\mathcal{B}$-presheaves is a weak equivalence if for every $\mathbf{B} \in \mathcal{B}$, $\mathfrak{f}_{*}: \mathfrak{X}\{\mathbf{B}\} \rightarrow \mathfrak{Y}\{\mathbf{B}\} \quad$ is a weak equivalence in $\mathcal{C}$ (as in 1.1).

By a suitable left Bousfield localization of $\mathcal{S}_{*}^{\Theta_{\mathcal{B}}^{\text {op }}}$ and $\mathcal{S}_{*}^{\Theta^{\mathcal{A}}}$ we can obtain model categories for weak $\mathcal{B}$-mapping algebras and dual weak $\mathcal{A}$-mapping algebras (i.e., model structures on the (dual) presheaf category in which the latter are the fibrant objects). However, since we cannot guarantee that these localized model structures are right proper (cf. [H, 3.4.4]), they will not be used in this paper.

1.21. Remark. Note that since we assumed the objects of $\boldsymbol{\Theta}_{\mathcal{B}}$ are cofibrant, when $\mathbf{Y}$ is fibrant the realizable $\mathcal{B}$-presheaf $\mathfrak{M}_{\mathcal{B}} \mathbf{Y}$ will be fibrant (that is, $\mathfrak{M}_{\mathcal{B}} \mathbf{Y}\{\mathbf{B}\}$ is a Kan complex for each $\left.\mathbf{B} \in \mathbf{\Theta}_{\mathcal{B}}\right)$. Similarly, for $\mathcal{A}$-dual presheaves, $\mathfrak{M}^{\mathcal{A}} \mathbf{Y}$ is fibrant if $\mathbf{Y}$ is cofibrant in $\mathcal{C}$.

1.22. Model categories of simplicial $\Pi$-algebras. Because both $\Pi_{\mathcal{B}}$-algebras (\$1.11) and $\Pi^{\mathcal{A}}$-algebras (\$1.18) are universal algebras in the sense of [Mc, VI, §8] having an underlying graded group structure, there is a model category structure on both the category $\Pi_{\mathcal{B}-A l g}{ }^{\Delta^{\text {op }}}$ of simplicial $\Pi_{\mathcal{B}}$-algebras and the category $\Pi^{\mathcal{A}}-\mathrm{Alg}^{\Delta^{\mathrm{op}}}$ of simplicial $\Pi^{\mathcal{A}}$-algebras. In both cases a map $f: U_{\bullet} \rightarrow V_{\bullet}$ of simplicial $\Pi$-algebras is a weak equivalence (respectively, fibration) if and only if the map $f_{*}: U_{\bullet}\{\mathbf{B}\} \rightarrow$ $\mathbf{W} \bullet\{\mathbf{B}\}$ is a weak equivalence (respectively, fibration) of simplicial groups for each $\mathbf{B} \in \operatorname{Obj} \boldsymbol{\Theta}$. The cofibrant objects are retracts of free simplicial objects.

1.23. Truncating mapping algebras. Fix $n \geq 0$. Given a $\mathcal{B}$-presheaf $\mathfrak{X}: \boldsymbol{\Theta}_{\mathcal{B}}^{\text {op }} \rightarrow$ $\mathcal{S}_{*}$, we may post-compose $\mathfrak{X}$ with the $n$-th Postnikov section functor $P^{n}: \mathcal{S}_{*} \rightarrow \mathcal{S}_{[n]}$ to obtain a new $\mathcal{B}$-presheaf $P^{n} \mathfrak{X}$, which we now think of as a continuous functor on $P^{n} \boldsymbol{\Theta}_{\mathcal{B}} \quad$ - that is, the sketch enriched in $\mathcal{S}_{[n]}$ obtained from $\boldsymbol{\Theta}_{\mathcal{B}}$ by applying $P^{n} \quad$ to each mapping space.

This is simplest to describe when $\mathfrak{X}$ is fibrant (cf. 1.21 ), since then we can use the $(n+1)$-coskeleton functor $\operatorname{csk}_{n+1}: \mathcal{S}_{*} \rightarrow \mathcal{S}_{*} \quad$ (which strictly preserves products) as our model for $P^{n}$. Note that the mapping spaces of $\boldsymbol{\Theta}_{\mathcal{B}}$ are always fibrant, since we assumed that all its objects are both fibrant and cofibrant. In the general case, we must first apply a fibrant replacement functor to $\mathfrak{X}$ in the model category $\mathcal{S}_{*}^{\boldsymbol{\Theta}_{\mathcal{B}}^{\text {op }}}$ of 1.20 .

The category of $n$-truncated $\mathcal{B}$-presheaves will be denoted by $\mathcal{S}_{[n]}^{\Theta_{\mathcal{B}}^{\text {op }}} \subset \mathcal{S}_{*}^{\Theta_{\mathcal{B}}^{\text {op }}}$, with the truncation functor $\gamma_{[n]}: \mathcal{S}_{*}^{\Theta_{\mathcal{B}}^{\text {op }}} \rightarrow \mathcal{S}_{[n]}^{\Theta_{\mathrm{B}}^{\text {op }}}$.

If $\mathfrak{X}$ is a (strict or weak) $\mathcal{B}$-mapping algebra, this usually will not be true of $P^{n} \mathfrak{X}$, since in general

$$
P^{n} \operatorname{map}(\Sigma \mathbf{B}, \mathbf{Y}) \simeq P^{n} \Omega \operatorname{map}(\mathbf{B}, \mathbf{Y}) \not P^{n-1} \Omega \operatorname{map}(\mathbf{B}, \mathbf{Y}) \simeq \Omega P^{n} \operatorname{map}(\mathbf{B}, \mathbf{Y}) .
$$

Thus we must modify Definition 1.1 as follows, assuming for simplicity that the category $\mathcal{F}$ consists as above of the inclusions $i_{0}, i_{1}: \Delta[0] \hookrightarrow \Delta[1]$, and the cocone collection $\mathcal{E}$ contains all coproducts of cardinality $<\lambda$ for some fixed limit cardinal $\lambda$, and the pushout squares (1.5):

(1) An $n$-truncated strict $\mathcal{B}$-mapping algebra is an $n$-truncated $\mathcal{B}$-presheaf $\mathfrak{X}$ for which the natural maps of (1.2) are isomorphisms for all $\mathbf{B} \in \boldsymbol{\Theta}_{\mathcal{B}}, \quad K \in \mathcal{F}$, and diagrams $I$ in $\mathcal{E}$, except for the right hand square in (1.6), where we 
have instead:

$$
\mathfrak{X}\{\Sigma \mathbf{B}\} \rightarrow P^{n-1} \mathfrak{X}\{\Sigma \mathbf{B}\} \cong P^{n-1} \Omega \mathfrak{X}\{\mathbf{B}\} \cong \Omega \mathfrak{X}\{\mathbf{B}\}
$$

where the first and last maps are the standard fibrations, the middle map is the natural map of (1.2), and $\Omega \mathfrak{X}\{\mathbf{B}\}$ is an $(n-1)$-type by assumption, with the last map an isomorphism.

The full subcategory of $n$-truncated strict $\mathcal{B}$-mapping algebras will be denoted by $\operatorname{sMap}_{\mathcal{B}}^{n}$.

(2) An $n$-truncated weak $\mathcal{B}$-mapping algebra is an $n$-truncated $\mathcal{B}$-presheaf $\mathfrak{X}$ weakly equivalent to an $n$-truncated strict $\mathcal{B}$-mapping algebra. This implies that the maps of (1.2), and the two right maps in (1.25), are weak equivalences (see Remark 1.3). The full subcategory of $n$-truncated weak $\mathcal{B}$-mapping algebras will be denoted by $\operatorname{wMap}_{\mathcal{B}}^{n}$.

In particular, for any $\mathbf{Y} \in \mathcal{C}$ we have the associated realizable $n$-truncated strict $\mathcal{B}$-mapping algebra $P^{n} \mathfrak{M}_{\mathcal{B}} \mathbf{Y}$, which is free if $\mathbf{Y} \in \boldsymbol{\Theta}_{\mathcal{B}}$, and the analogue of Lemma 1.8 still holds. We define the $n$-truncated versions of $\mathcal{A}$-dual presheaves and (strict or weak) dual $\mathcal{A}$-mapping algebras dually.

\section{FACtoring FUnCtors through MAPPing ALGEBras}

The first step in our program is to show that suitable homotopy functors $\mathbf{T}$ : $\mathcal{C} \rightarrow \mathcal{D}$ factor up to weak equivalence through an appropriate category of mapping algebras: in other words, find an enriched sketch $\Theta_{\mathcal{B}}$ and a functor $\mathfrak{T}: \mathcal{S}_{*}^{\Theta_{\mathcal{B}}^{\text {op }}} \rightarrow \mathcal{D}$, equipped with a natural weak equivalence $\mathfrak{T} \circ \mathfrak{M}_{\mathcal{B}} \rightarrow \mathbf{T}$. In fact, $\mathfrak{T}$ need not be defined on all of $\mathcal{S}_{*}^{\Theta_{\mathcal{B}}^{\text {op }}}$; it suffices if it is defined on the subcategory sMap $_{\mathcal{B}}$ of strict $\mathcal{B}$-mapping algebras where $\mathfrak{M}_{\mathcal{B}}$ takes values.

Dually, we could try to find a dual enriched sketch $\boldsymbol{\Theta}^{\mathcal{A}}$ and a functor $\mathfrak{T}^{\prime}$ : $\operatorname{sMap}^{A} \rightarrow \mathcal{D}$ with a natural weak equivalence $\mathbf{T} \rightarrow \mathfrak{T}^{\prime} \circ \mathfrak{M}^{\mathcal{A}}$.

\section{A. Realizing mapping algebras}

The simplest way to define such a functor $\mathfrak{T}$ is in the case where every strict $\mathcal{B}$ mapping algebra $\mathfrak{X}$ is (functorially) realizable. Essentially, the only case where this is known to be true is when $\mathcal{C}=\operatorname{Top}_{0}$ and $\mathcal{B}=\left\{\mathbf{S}^{n}\right\}_{n=1}^{\infty}$. We briefly summarize the construction of [BB2, §9] (based on that of [Sto, §2]):

2.1. The Stover construction. Recall that for a pointed Kan complex $K \in \mathcal{S}_{*}$, the path space $P K$ is given by $(P K)_{n}:=\left\{x \in K_{n+1}: d_{1} \ldots d_{n+1} x=*\right\}$, with re-indexed face and degeneracy maps, and the universal fibration $p: P K \rightarrow K$ is induced by $d_{0}$ (cf. [Mo]). Thus when $K$ is a simplicial group, the map on 0-simplices $p_{0}:(P K)_{0} \rightarrow K_{0} \quad$ suffices to compute $\pi_{0} K$. We therefore choose the category $\mathcal{G}=\mathrm{Gp}^{\Delta^{\mathrm{op}}}$ of simplicial groups as our model $\mathcal{C}$ for the homotopy theory of pointed connected spaces, and set $\mathcal{B}:=\left\{\mathcal{S}^{n}\right\}_{n=1}^{\infty} \quad$ (where $\mathcal{S}^{n}:=F \mathbf{S}^{n-1}$, as a free simplicial group, is a strict cogroup object modelling the $n$-sphere in $\mathcal{G}$ ). For any limit cardinal $\lambda$, the resulting enriched sketch $\Theta_{\mathcal{B}}=\Theta_{\mathcal{B}}^{\lambda}$ then has a strict mapping algebra functor $\mathfrak{M}_{\mathcal{B}}: \mathcal{G} \rightarrow \operatorname{sMap}_{\mathcal{B}}$ with each $\mathfrak{M}_{\mathcal{B}} \mathbf{Y}\{\mathbf{B}\}$ a simplicial group (though the structure maps are just maps of pointed simplicial sets, in general). 
2.2. Definition. Let $\Gamma:=\mathbf{1}^{\mathbb{N}}$ be the category consisting of a countable collection of arrows, indexed by the objects of $\mathcal{B}$, and $\operatorname{Set}_{*}^{\Gamma}$ the category of $\Gamma$-indexed diagrams $\Phi:=\left(\phi_{n}: E_{n} \rightarrow F_{n}\right)_{n \in \mathbb{N}}$ in pointed sets, called arrow sets. We have a forgetful functor $\rho: \operatorname{sMap}_{\mathcal{B}} \rightarrow \operatorname{Set}_{*}^{\Gamma}$, with $(\rho \mathfrak{X})_{n}=\left(p_{0}:\left(P \mathfrak{X}\left\{\mathcal{S}^{n}\right\}_{0} \rightarrow\left(\mathfrak{X}\left\{\mathcal{S}^{n}\right\}\right)_{0}\right)\right.$. In fact, $\rho \mathfrak{X}$ is defined for any presheaf $\mathfrak{X}: \Theta_{\mathcal{B}}^{\text {op }} \rightarrow \mathcal{S}_{*}$, but we are only interested in the composite $\mathcal{R}_{\mathcal{B}}:=\rho \mathfrak{M}_{\mathcal{B}}: \mathcal{G} \rightarrow \operatorname{Set}_{*}^{\Gamma}$. This has a left adjoint $\mathcal{L}_{\mathcal{B}}:$ Set $_{*}^{\Gamma} \rightarrow \mathcal{G}$, which assigns to an arrow set $\Phi=\left(\phi_{n}: E_{n} \rightarrow F_{n}\right)_{n \in \mathbb{N}}$ the coproduct

$$
\mathcal{L}_{\mathcal{B}} \Phi:=\coprod_{n \in \mathbb{N}} \coprod_{f \in F_{n}} Q_{(f)},
$$

where we define $Q_{(f)}$ for $f \in F_{n}$ as follows:

(a) If $* \neq f \in \operatorname{Im} \phi_{n}$, then $Q_{(f)}$ is defined by the pushout square

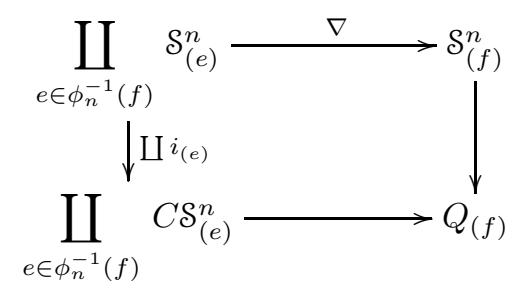

in $\mathcal{G}$ (where $i: \mathcal{S}^{n} \rightarrow C \mathcal{S}^{n}$ is the inclusion into the cone, and $\nabla$ is the fold map).

(b) If $f \notin \operatorname{Im} \phi_{n}$, we set $Q_{(f)}:=\mathcal{S}^{n}$.

(c) If $f=*$, we set

$$
Q_{(f)}:=\coprod_{* \neq e \in \phi_{n}^{-1}(*)} \Sigma \mathcal{S}_{(e)}^{n} .
$$

Compare [BS2, §2] and [Sto, §2], where the comonad $\mathcal{V}_{\mathcal{B}}=\mathcal{L}_{\mathcal{B}} \mathcal{R}_{\mathcal{B}}: \mathcal{G} \rightarrow \mathcal{G}$ (or rather, its analogue for $\mathrm{Top}_{0}$ ) was used to construct functorial resolutions of pointed connected spaces by wedges of spheres.

Note that each $Q_{(f)}$, and thus $\mathcal{L}_{\mathcal{B}} \Phi$, is a strict cogroup object in $\mathcal{G}$ (fibrant and cofibrant) of the homotopy type of a wedge of spheres. If $\lambda$ is any limit cardinal, we define a $\lambda$-Stover space to be any pushout of the form (2.4), with $\phi_{n}^{-1}(f)$ replaced by any set $T$ of cardinality $<\lambda$. Let $\boldsymbol{\Theta}_{\mathrm{St}}=\boldsymbol{\Theta}_{\mathrm{St}}^{\lambda}$ denote a skeleton of the sub-simplicial category of $\mathcal{G}$ whose objects are coproducts of $\lambda$-Stover spaces over indexing sets of cardinality $<\lambda$. This is an enriched sketch, with $\mathcal{F}$ as in 1.4 and $\mathcal{E}$ consisting of the coproducts of cardinality $<\lambda$ in $\boldsymbol{\Theta}_{\mathrm{St}}$, together with the pushout squares of (1.5) and (2.4). The category of the corresponding strict mapping algebras, called strict Stover mapping algebras, will be denoted by $\mathrm{sMap}_{\mathrm{St}}$, with $\mathfrak{M}_{\mathrm{St}}: \mathcal{G} \rightarrow \mathrm{sMap}_{\mathrm{St}}$ the strict Stover mapping algebra functor.

2.5. The algebra structure. Since each sphere $\mathcal{S}^{n} \in \mathcal{G}$ is in particular a Stover space, $\boldsymbol{\Theta}_{\mathcal{B}}=\boldsymbol{\Theta}_{\mathcal{B}}^{\lambda}$ is a full simplicial subcategory of $\boldsymbol{\Theta}_{\mathrm{St}}=\boldsymbol{\Theta}_{\mathrm{St}}^{\lambda}$, with $\iota: \boldsymbol{\Theta}_{\mathcal{B}} \hookrightarrow \boldsymbol{\Theta}_{\mathrm{St}}$ the inclusion, inducing the restriction $\iota^{*}: \mathrm{sMap}_{\mathrm{St}} \rightarrow \operatorname{sMap}_{\mathcal{B}}$. Write $\hat{\rho}: \operatorname{sMap}_{\mathrm{St}} \rightarrow$ $\mathrm{Set}_{*}^{\Gamma}$ for the composite $\rho \circ \iota^{*}$.

We claim that for every strict Stover mapping algebra $\mathfrak{X}$, the arrow set $\rho \mathfrak{X}$ has a natural $\mathcal{T}_{\mathcal{B}}$-algebra structure map $h: \mathcal{T}_{\mathcal{B}} \rho \mathfrak{X} \rightarrow \rho \mathfrak{X}$ for the monad $\mathcal{T}_{\mathcal{B}}=$ 
$\mathcal{R}_{\mathcal{B}} \mathcal{L}_{\mathcal{B}}: \operatorname{Set}_{*}^{\Gamma} \rightarrow \operatorname{Set}_{*}^{\Gamma} \quad($ see $[$ Bor, $\S 4.1])$. If we set $\mathcal{K}:=\mathcal{L}_{\mathcal{B}} \circ \hat{\rho}: \operatorname{sMap}_{\mathrm{St}} \rightarrow \mathcal{G}$ and $\mathcal{V}_{\mathcal{B}}:=\mathfrak{M}_{\mathrm{St}} \circ \mathcal{K}$, we may display the various functors defined in the following commuting diagram:

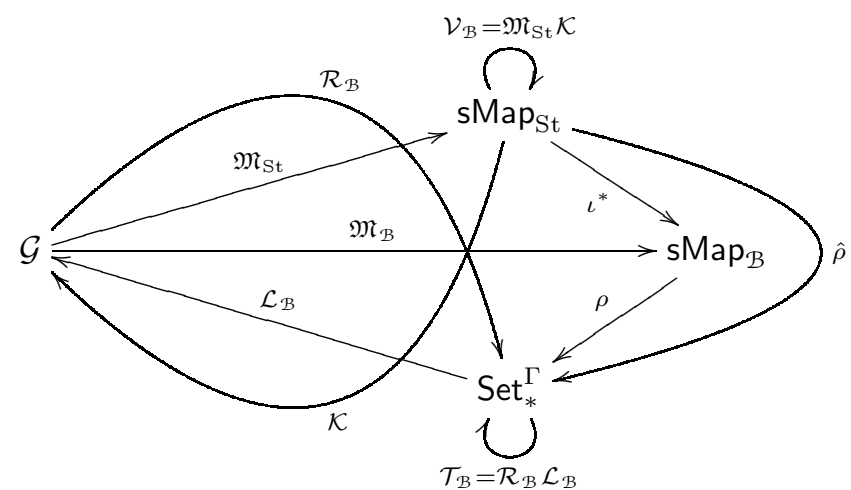

In this setting we have a stronger statement (cf. [BB2, 9.19]):

2.7. Lemma. Every strict Stover mapping algebra $\mathfrak{X}$ has a natural map $\xi_{\mathfrak{X}}: \mathcal{V}_{\mathcal{B}} \mathfrak{X} \rightarrow$ $\mathfrak{X}$ making the following diagram

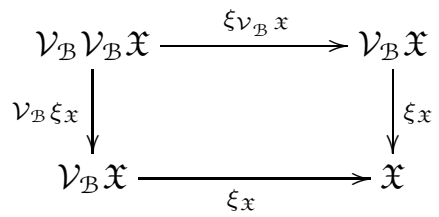

commute in $\mathrm{sMap}_{\mathrm{St}}$, where $\xi_{\mathcal{V}_{\mathfrak{B}} \mathfrak{X}}=\mathfrak{M}_{\mathrm{St}} \varepsilon_{\mathcal{K} \mathfrak{X}}$ for $\varepsilon: \mathcal{K} \mathfrak{M}_{\mathrm{St}} \rightarrow \mathrm{Id}$ the counit of the comonad $\mathcal{L}_{\mathcal{B}} \mathcal{R}_{\mathcal{B}}$.

The structure map $h: \mathcal{T}_{\mathcal{B}} \hat{\rho} \mathfrak{X} \rightarrow \hat{\rho} \mathfrak{X}$ is then given by $\hat{\rho}\left(\xi_{\mathfrak{X}}\right)$, since $\mathcal{T}_{\mathcal{B}} \circ \hat{\rho}=\hat{\rho} \circ \mathcal{V}_{\mathcal{B}}$ (see (2.61)).

Proof. Let $\mathcal{D}^{i}$ denote either $\mathcal{S}^{n_{i}}$ or $C \mathcal{S}^{n_{i}}$ in $\mathcal{G}$.

(a) Recall that $\mathcal{K} \mathfrak{X}$ is defined for any strict Stover mapping algebra $\mathfrak{X}$ by the colimit (2.4), which we may write as $\operatorname{colim}_{i} \mathcal{D}_{f_{i}}^{i}$, where $f_{i} \in \mathfrak{X}\left\{\mathcal{D}^{i}\right\}_{0}$. Since $\mathcal{K} \mathfrak{X} \in \Theta_{\mathrm{St}}$, the strict Stover mapping algebra $\mathcal{V}_{\mathcal{B}} \mathfrak{X}$ is free, so to define the algebra structure map $\xi_{\mathfrak{X}}: \mathcal{V}_{\mathcal{B}} \mathfrak{X} \rightarrow \mathfrak{X}$ we need only specify $\xi_{\mathfrak{X}}\left(\operatorname{Id}_{\mathcal{K} \mathfrak{X}}\right) \in \mathfrak{X}\{\mathcal{K} \mathfrak{X}\}_{0}$. But $\mathfrak{X}$ takes the colimit of (2.4) to a limit, so $\xi_{\mathfrak{X}}\left(\operatorname{Id}_{\mathcal{K} \mathfrak{X}}\right)$ is determined by the elements $f_{i} \in \mathfrak{X}\left\{\mathcal{D}^{i}\right\}_{0}$. We therefore write $\xi_{\mathfrak{X}}\left(\operatorname{Id}_{\mathcal{K} \mathfrak{X}}\right)=\bigoplus_{i} f_{i}$, where $\oplus$ indicates that we are using the duality (1.2) between the colimits and the limits.

(b) Similarly, for any $Y \in \mathcal{G}$ we have $\mathcal{K} \mathfrak{M}_{\mathrm{St}} Y=\operatorname{colim}_{j} \mathcal{D}_{g_{j}}^{j}$. The counit $\varepsilon_{Y}$ : $\mathcal{K} \mathfrak{M}_{\mathrm{St}} Y \rightarrow Y$ is again determined by the indexing maps as $\varepsilon_{Y}=\operatorname{colim}_{j} g_{j}$, with the induced map $\mathfrak{M}_{\mathrm{St}} \varepsilon_{Y}: \mathcal{V} \mathfrak{M}_{\mathrm{St}} Y \rightarrow \mathfrak{M}_{\mathrm{St}} Y$ sending $\operatorname{Id}_{\mathcal{K}_{\mathfrak{M}_{\mathrm{St}}} Y}$ in $\mathfrak{M}_{\mathrm{St}} \mathcal{K} \mathfrak{M}_{\mathrm{St}} Y\left\{\mathcal{K} \mathfrak{M}_{\mathrm{St}} Y\right\}_{0} \quad$ to $\left[\operatorname{colim}_{j} g_{j}\right] \quad$ in $\mathfrak{M}_{\mathrm{St}} Y\left\{\mathcal{K} \mathfrak{M}_{\mathrm{St}} Y\right\}_{0}$.

Thus when $\mathfrak{X}=\mathfrak{M}_{\mathrm{St}} Y$, the map $\xi_{\mathfrak{X}}$ sends $\operatorname{Id}_{\mathcal{K} \mathfrak{M}_{\mathrm{St}} Y}$ to $\varepsilon_{Y}=\operatorname{colim}_{j} g_{j}$ in $\mathfrak{X}\left\{\mathcal{K} \mathfrak{M}_{\mathrm{St}} Y\right\}_{0}=\operatorname{map}\left(\mathcal{K} \mathfrak{M}_{\mathrm{St}} Y, Y\right)_{0}$. This means that $\xi_{\mathfrak{M}_{\mathrm{St}} Y}=\mathfrak{M}_{\mathrm{St}} \varepsilon_{Y} ;$ in particular, the top horizontal map in (2.8) is $\xi_{\mathcal{V}_{\mathcal{B}} \mathfrak{X}}$. 
(c) To evaluate the top right composite $\varphi:=\xi_{\mathfrak{X}} \circ \mathfrak{M}_{\mathrm{St}} \varepsilon_{\mathcal{K} \mathfrak{X}}: \mathcal{V}_{\mathcal{B}} \mathcal{V}_{\mathcal{B}} \mathfrak{X} \rightarrow \mathfrak{X}$, note that $\mathcal{V}_{\mathcal{B}} \mathcal{V}_{\mathcal{B}} \mathfrak{X}$ is free on $\mathcal{K} \mathcal{V}_{\mathcal{B}} \mathfrak{X}$, so we need only specify $\varphi\left(\operatorname{Id}_{\mathcal{K}} \mathcal{V}_{\mathcal{B}} \mathfrak{X}\right)$ in $\mathfrak{X}\left\{\mathcal{K} \mathcal{V}_{\mathcal{B}} \mathfrak{X}\right\}$. Since $\xi_{\mathfrak{X}}$ is a map of strict Stover mapping algebras, it sends $\left[\operatorname{colim}_{j} g_{j}\right] \in \mathcal{V}_{\mathcal{B}} \mathfrak{X}\left\{\mathcal{K} \mathcal{V}_{\mathcal{B}} \mathfrak{X}\right\}_{0} \quad($ for $Y:=\mathcal{K} \mathfrak{X} \quad$ in (b) above) to

$$
\left[\perp_{j} g_{j}\right]^{*}\left(\xi_{\mathfrak{X}}\left(\operatorname{Id}_{\mathcal{K} \mathfrak{X}}\right)=\top_{j} g_{j}^{*}\left(\bigoplus_{i} f_{i}\right) \text { in } \mathfrak{X}\left\{\mathcal{K} \mathcal{V}_{\mathcal{B}} \mathfrak{X}\right\}_{0}\right.
$$

(d) Since $\mathcal{V}_{\mathcal{B}} \mathcal{V}_{\mathcal{B}} \mathfrak{X}$ is free, the map $\mathcal{V}_{\mathcal{B}} \xi_{\mathfrak{X}}: \mathcal{V}_{\mathcal{B}} \mathcal{V}_{\mathcal{B}} \mathfrak{X} \rightarrow \mathcal{V}_{\mathcal{B}} \mathfrak{X}$ is determined by where it sends $\operatorname{Id}_{\mathcal{K} \mathcal{V}_{\mathcal{B}} \mathfrak{X}}$ in $\mathcal{V}_{\mathcal{B}} \mathfrak{X}\left\{\mathcal{K} \mathcal{V}_{\mathcal{B}} \mathfrak{X}\right\}_{0}=\operatorname{map}\left(\mathcal{K} \mathcal{V}_{\mathcal{B}} \mathfrak{X}, \mathcal{K} \mathfrak{X}\right)_{0}$, namely, to $\mathcal{K} \xi_{\mathfrak{X}}: \mathcal{K} \mathcal{V}_{\mathcal{B}} \mathfrak{X} \rightarrow \mathcal{K} \mathfrak{X}$. Since $\mathcal{K} \mathcal{V}_{\mathcal{B}} \mathfrak{X}=\operatorname{colim}_{j} \mathcal{D}_{g_{j}}^{j} \quad$ where the colimit is over all maps $g_{j}: \mathcal{D}^{j} \rightarrow \mathcal{K} \mathfrak{X}$, we see from the description of $\xi_{\mathfrak{X}}$ above (and the construction of $\mathcal{K}$ ) that $\mathcal{K} \xi_{\mathfrak{X}} \quad$ sends $\mathcal{D}_{g_{j}}^{j}$ to the copy of $\mathcal{D}^{j}$ in the colimit defining $\mathcal{K} \mathfrak{X}$ indexed by

$$
\xi_{\mathfrak{X}}\left(g_{j}\right)=\xi_{\mathfrak{X}}\left(g_{j}^{*}\left(\operatorname{Id}_{\mathcal{K} \mathfrak{X}}\right)\right)=g_{j}^{*}\left(\xi_{\mathfrak{X}}\left(\operatorname{Id}_{\mathcal{K} \mathfrak{X}}\right)\right)=g_{j}^{*}\left(\bigoplus_{i} f_{i}\right)
$$

in $\mathfrak{X}\left\{\mathcal{D}^{j}\right\}_{0}$, where $\xi_{\mathfrak{X}}\left(\operatorname{Id}_{\mathcal{K} \mathfrak{X}}\right)=\bigoplus_{i} f_{i}$ by $(\mathrm{a})$.

Thus the element $\xi_{\mathfrak{X}}\left(\mathcal{V}_{\mathcal{B}} \xi_{\mathfrak{X}}\left(\operatorname{Id}_{\left.\left.\mathcal{K} \mathcal{V}_{\mathcal{B}} \mathfrak{X}\right)\right)}\right.\right.$ in $\mathfrak{X}\left\{\mathcal{K} \mathcal{V}_{\mathcal{B}} \mathfrak{X}\right\}_{0}$ is determined by the fact that $\mathfrak{X}$ takes the colimit $\operatorname{colim}_{j} \mathcal{D}_{g_{j}}^{j}$ defining $\mathcal{K} \mathcal{V}_{\mathcal{B}} \mathfrak{X}$ to a limit, namely:

$$
\xi_{\mathfrak{X}}\left(\mathcal{V}_{\mathcal{B}} \xi_{\mathfrak{X}}\left(\operatorname{Id}_{\mathcal{K} \mathcal{V}_{\mathcal{B}} \mathfrak{X}}\right)\right)=\xi_{\mathfrak{X}}\left(\mathcal{K} \xi_{\mathfrak{X}}\right)=\xi_{\mathfrak{X}}\left(\perp_{j} g_{j}\right)=\top_{j} \xi_{\mathfrak{X}}\left(g_{j}\right)=\top_{j} g_{j}^{*}\left(\bigoplus_{i} f_{i}\right)
$$

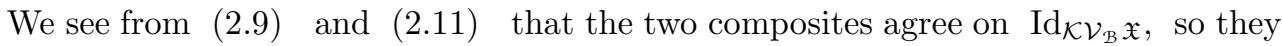
are equal.

2.12. The resolution model category of simplicial presheaves. For any set $\mathcal{B} \subset \mathcal{C}$ as in $₫ 1.1$ consider the category $\left(\mathcal{S}_{*}^{\Theta_{\mathcal{B}}^{\mathrm{op}}}\right)^{\Delta^{\mathrm{op}}}=\mathcal{S}_{*}^{\Theta_{\mathcal{B}}^{\mathrm{op}} \times \Delta^{\mathrm{op}}} \quad$ of simplicial $\mathcal{B}$-presheaves - that is, simplicial objects in the category of $\mathcal{B}$-presheaves. As noted in 11.20 , the $\mathcal{B}$-presheaf category $\mathcal{S}_{*}^{\Theta_{\mathcal{B}}^{\text {op }}}$ has a proper simplicial model category structure. Moreover, the objects of $\mathcal{B}$ are homotopy cogroup objects in $\mathcal{C}$, as are their colimits under $\mathcal{E}$ as in 1.4 . Therefore, as in [J, §2], there is a resolution model category structure on $\mathcal{S}_{*}^{\Theta_{\mathcal{B}}^{\mathrm{op}} \times \Delta^{\mathrm{op}}}$, for which the projectives of $\mathcal{S}_{*}^{\Theta_{\mathcal{B}}^{\mathrm{op}}}$ are the free strict $\mathcal{B}$-mapping algebras. A map $\mathfrak{f}: \mathfrak{V}_{\bullet} \rightarrow \mathfrak{W}_{\bullet} \quad$ of simplicial $\mathcal{B}$-presheaves is a weak equivalence in this model category if and only if it is an $E^{2}$-equivalence - that is, if for each $\mathbf{B} \in \boldsymbol{\Theta}_{\mathcal{B}}$ and $t, s, \geq 0$, the map $\mathfrak{f}_{*}: \pi_{t}^{h} \pi_{s}^{v} \mathfrak{V}_{\bullet}\{\mathbf{B}\} \rightarrow \pi_{t}^{h} \pi_{s}^{v} \mathfrak{W}_{\bullet}\{\mathbf{B}\} \quad$ is an isomorphism (the terminology comes from the $E^{2}$-term of the homotopy spectral sequence of a simplicial space - cf. [DKS1]).

Note that if a simplicial presheaf $\mathfrak{W}_{\bullet}$ is cofibrant, each $\mathfrak{W}_{n}$ is weakly equivalent to a coproduct of free strict $\mathcal{B}$-mapping algebras, so in particular it is a weak $\mathcal{B}$ mapping algebra. Moreover, in order for $\mathfrak{W}$ • to be a resolution of a weak $\mathcal{B}$ mapping algebra $\mathfrak{X}$, in particular $\pi_{0} \mathfrak{W} \bullet$ must be a resolution of $\pi_{0} \mathfrak{X}$ in the model category of simplicial $\Pi_{\mathcal{B}}$-algebras (see $§ 1.22$ ), so that the augmented simplicial group $\pi_{0} \mathfrak{W} \bullet\{\mathbf{B}\} \rightarrow \pi_{0} \mathfrak{X}\{\mathbf{B}\} \quad$ is weakly contractible for any $\mathbf{B} \in \mathbf{\Theta}_{\mathcal{B}}$.

We observe also that $\mathcal{S}_{*}^{\Theta_{\mathcal{B}}^{\mathrm{op}} \times \Delta^{\text {op }}}$ has a Reedy model category structure, with weak equivalences and fibrations defined at each simplicial space $\mathfrak{V}_{\bullet}\{\mathbf{B}\}$ for every $\mathbf{B} \in \boldsymbol{\Theta}_{\mathcal{B}} \quad($ see $[\mathrm{H}, \S 15.3])$. 
Since $P^{n}$ is a nullification, $\mathcal{S}_{[n]}$ is still right proper (see [Bou3, Theorem 9.9], so we have an analogous resolution model category structure on the category $\mathcal{S}_{[n]}^{\boldsymbol{\Theta}_{\mathrm{B}}^{\text {op }}}$ of $n$-truncated simplicial $\mathcal{B}$-presheaves (\$1.23).

We deduce the following enhancement of [BB2, Proposition 9.23]:

2.13. Theorem. There is a realization functor $N: \mathrm{sMap}_{\mathrm{St}} \rightarrow \mathcal{G}$, equipped with natural weak equivalences $\theta: N \circ \mathfrak{M}_{\mathrm{St}} \rightarrow \operatorname{Id}_{\mathcal{G}} \quad$ and $\zeta: \mathfrak{M}_{\mathrm{St}} \circ N \rightarrow \operatorname{Id}_{\mathrm{sMap}_{\mathrm{St}}}$.

Proof. Given a strict Stover mapping algebra $\mathfrak{X} \in \mathrm{sMap}_{\mathrm{St}}$, iterating the comonad $\mathcal{U}:=\mathcal{L}_{\mathcal{B}} \mathcal{R}_{\mathcal{B}}: \mathcal{G} \rightarrow \mathcal{G}$ on $Y:=\mathcal{K} \mathfrak{X}=\mathcal{L}_{\mathcal{B}} \hat{\rho} \mathfrak{X}$ yields an augmented simplicial space Z. $\rightarrow Y$ with $\mathbf{Z}_{n}:=\mathcal{U}^{n+1} Y$ and $d_{i}: \mathbf{Z}_{n} \rightarrow \mathbf{Z}_{n-1}$ given by as usual by $\mathcal{U}^{i} \varepsilon_{\mathcal{U}^{n-i} Y}$ (cf. [W, $\S 8.6 .4]$ ).

Since by (2.6) $\mathcal{U}=\mathcal{L}_{\mathcal{B}} \mathcal{R}_{\mathcal{B}}=\mathcal{K} \mathfrak{M}_{\mathrm{St}}$ and $\mathcal{V}_{\mathcal{B}}=\mathfrak{M}_{\mathrm{St}} \mathcal{K}$, we have a simplicial strict Stover mapping algebra $\mathfrak{W}_{\bullet}=\mathfrak{M}_{\mathrm{St}} \mathbf{Z}_{\bullet}$, which augments to $\mathfrak{X}$ via $\xi_{\mathfrak{X}}: \mathfrak{M}_{\mathrm{St}} Y=$ $\mathcal{V}_{\mathcal{B}} \mathfrak{X} \rightarrow \mathfrak{X}$, by Lemma 2.7 Applying $\mathcal{K}$ to $\mathfrak{W}_{\bullet} \rightarrow \mathfrak{X}$ recovers $\mathbf{Z}_{\bullet} \rightarrow Y$, but now with an extra degeneracy in each simplicial dimension coming from the unit $\eta: \mathrm{Id} \rightarrow \mathcal{T}_{\mathcal{B}}=\mathcal{R}_{\mathcal{B}} \mathcal{L}_{\mathcal{B}}$ of the corresponding monad, as well as an extra face map, obtained by iterating $\mathcal{U}$ on $\mathcal{K} \xi_{\mathfrak{X}}: \mathcal{K} \mathcal{V}_{\mathcal{B}} \mathfrak{X}=\mathbf{Z}_{1} \rightarrow \mathcal{K} \mathfrak{X}=\mathbf{Z}_{0}$. By commutativity of (2.8), we see that $\mathbf{Z}_{\bullet} \rightarrow Y$ is in fact the décalage of a simplicial space $\mathbf{X}_{\bullet}$ (see [I]). Moreover, applying $\mathfrak{M}_{\mathrm{St}}$ to $\mathbf{X}_{\bullet}$ yields an augmented (free) simplicial strict Stover mapping algebra $\mathfrak{M}_{\mathrm{St}} \mathbf{X} \bullet \rightarrow \mathfrak{X}$ which is a resolution of $\mathfrak{X}$ in the sense of \$2.12.

This shows that the Quillen-Bousfield-Friedlander spectral sequence for $\mathbf{X}_{\bullet}$ (see Q1 and [BF, Theorem B.5]) collapses, so that $N \mathfrak{X}:=\left\|\mathbf{X}_{\bullet}\right\|$ realizes $\mathfrak{X}$ up to weak equivalence. Noting that $\mathbf{X}_{\bullet}$ is obtained by applying $\mathcal{K}$ to $\zeta_{0}: \mathfrak{M}_{\mathrm{St}} \mathbf{X}_{\bullet} \rightarrow \mathfrak{X}$, and that $\mathfrak{M}_{\mathrm{St}} \mathbf{X}_{\bullet}$ is constructed by iterating $\mathcal{V}_{\mathcal{B}}$ on $\mathfrak{X}$ (together with $\xi_{\mathfrak{X}}$ ), we have described a functorial procedure for realizing any strict Stover mapping algebra $\mathfrak{X}$. The natural weak equivalence $\zeta$ is induced by the augmentation $\zeta_{0}$, while $\theta$ comes from the counit of the Stover comonad.

2.14. Corollary. Any homotopy functor $\mathbf{T}: \mathcal{G} \rightarrow \mathcal{D}$ to a model category $\mathcal{D}$ induces a functor $\mathfrak{T}:=\mathbf{T} \circ N: \mathrm{sMap}_{\mathrm{St}} \rightarrow \mathcal{D}$ equipped with a natural weak equivalence $\vartheta=\mathbf{T} \theta: \mathfrak{T} \circ \mathfrak{M}_{\mathrm{St}} \rightarrow \mathbf{T}$.

\section{B. Realizing dual mapping algebras}

To dualize the results of $₫ 2 \mathrm{~A}$, we want a setting where every dual strict $\mathcal{A}$-mapping algebra $\mathfrak{X}$ is functorially realizable. Again we have only one case where this is known to be true, when $\mathcal{C}=\mathcal{S}^{\text {red }}$ (or similar model categories for pointed connected spaces) and $\mathcal{A}$ consists of certain simplicial $R$-modules for some commutative ring $R$.

2.15. Definition. In general, we must include in the corresponding enriched sketch $\Theta^{\mathcal{A}}$ all $R$-module GEMs up to a certain cardinality. In particular, when $\mathcal{C}=\mathcal{S}^{\text {red }}$ we let $\Theta^{R}=\Theta_{\lambda}^{R}:=s \mathcal{M}_{\lambda}^{R}$, be the full subsimplicial category of $\mathcal{C}$ consisting of all simplicial $R$-modules of cardinality $<\lambda$, for some limit cardinal $\lambda$ (determined as in [BS2, §3.B]). The corresponding dual mapping algebras will be called dual strict $R$ mapping algebras (or R-mapping algebras, for short), and the category of such will be denoted by $\mathrm{sMap}^{R}$, with $\mathfrak{M}^{R}: \mathcal{C}^{\mathrm{op}} \rightarrow \mathrm{sMap}^{R}$ the realizable $R$-mapping algebras. 
2.16. The dual Stover construction. As in 2.1, we have a forgetful functor $\rho: \mathrm{sMap}^{R} \rightarrow\left(\operatorname{Set}_{*}^{\Gamma}\right)^{\text {op }}$, with $(\rho \mathfrak{X})_{n}=\left(p_{0}:\left(P \mathfrak{X}\{K(R, n)\}_{0} \rightarrow(\mathfrak{X}\{K(R, n)\})_{0}\right)\right.$. The composite $\mathcal{L}^{R}:=\rho \mathfrak{M}^{R}: \mathcal{C} \rightarrow\left(\operatorname{Set}_{*}^{\Gamma}\right)^{\text {op }}$ has a right adjoint $\mathcal{R}^{R}:\left(\operatorname{Set}_{*}^{\Gamma}\right){ }^{\text {op }} \rightarrow \mathcal{C}$, with $\mathcal{R}^{R} \Phi:=\prod_{n \in \mathbb{N}} \prod_{f \in F_{n}} Q^{(f)} \quad$ for any arrow set $\Phi=\left(\phi_{n}: E_{n} \rightarrow F_{n}\right)_{n \in \mathbb{N}}$

When $R$ is a field, we define $Q^{(f)}$ for $f \in F_{n}$ by the pullback square

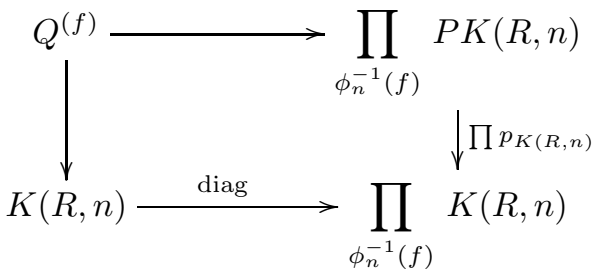

if $* \neq f \in \operatorname{Im} \phi_{n}$, while $Q^{(f)}:=K(R, n)$ if $f \notin \operatorname{Im} \phi_{n}$. If $\phi=*$, we set $Q^{(f)}:=\prod_{\phi_{n}^{-1}(*) \backslash\{*\}} \Omega K(R, n) \quad($ compare (2.4) $)$.

Again, for any limit cardinal $\Lambda$ we define a $\lambda$-R-Stover space to be any pullback of the form (2.17), with $\phi_{n}^{-1}(f)$ replaced by any set $T$ of cardinality $<\lambda$. When $R$ is not a field, we need to use the more complicated modified Stover construction of [BS2, §3.A] instead of the above.

We denote by $\Theta_{\lambda}^{\mathrm{St}, R}$ the corresponding dual enriched sketch, with $\mathcal{F}$ as in $\$ 1.14$ and $\mathcal{L}$ consisting of products of cardinality $<\lambda$ in $\Theta_{\lambda}^{\mathrm{St}, R}$, together with the pullback squares of (1.15) and (2.17). The category of the corresponding dual strict mapping algebras, called dual strict Stover mapping algebras, will be denoted by $\mathrm{sMap}^{\mathrm{St}, R}$, with $\mathfrak{M}^{\mathrm{St}, R}: \mathcal{C} \rightarrow \mathrm{sMap}^{\mathrm{St}, R}$ the dual strict Stover mapping algebra functor.

Since each $K(R, n)$ is in particular an $R$-Stover space, $\Theta_{\lambda}^{R}$ is a full simplicial subcategory of $\Theta_{\lambda}^{\mathrm{St}, R}$, with $\iota: \Theta_{\lambda}^{R} \hookrightarrow \Theta_{\lambda}^{\mathrm{St}, R}$ the inclusion, inducing the restriction $\iota^{*}: \mathrm{sMap}^{\mathrm{St}, R} \rightarrow \mathrm{sMap}^{R}$ as in 2.5 Writing $\mathcal{V}^{R}:=\mathfrak{M}^{\mathrm{St}, R} \circ \mathcal{R}^{R} \circ \rho \circ \iota^{*}: \mathrm{sMap}^{\mathrm{St}, R} \rightarrow$ $\mathrm{sMap}^{\mathrm{St}, R}$, we obtain the following categorical dual of Lemma 2.7 (compare [BS2, Proposition 2.19]):

2.18. Lemma. Every dual strict Stover mapping algebra $\mathfrak{X}$ has a natural map $\zeta_{\mathfrak{X}}$ : $\mathcal{V}^{R} \mathfrak{X} \rightarrow \mathfrak{X}$ making the following diagram commute in $\mathrm{sMap}^{\mathrm{St}, R}$ :

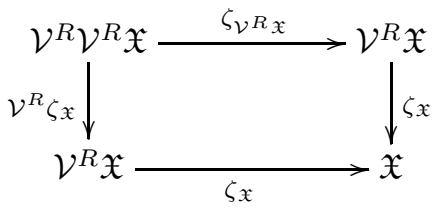

2.20. Definition. For any commutative ring $R$, we denote by $\mathcal{S}_{R}$ the full subcategory of $R$-good spaces in $\mathcal{S}_{*} \quad$ (cf. [BK1, I, §5.1]), and by $\mathrm{sMap}_{\mathrm{re}}^{\mathrm{St}, R}$ the full subcategory of $\mathrm{sMap}^{\mathrm{St}, R}$ consisting of those dual strict Stover mapping algebras which are weakly equivalent to $\mathfrak{M}^{\mathrm{St}, R} \mathbf{Y}$ for some $\mathbf{Y} \in \mathcal{S}_{R}$. These will be called weakly $R$-good dual strict Stover mapping algebras.

2.21. Remark. By [H, §15.3]), $\mathcal{S}_{*}^{\Theta^{\mathcal{A}} \times \Delta^{\text {op }}}$ and $\mathcal{S}_{[n]}^{\Theta^{\mathcal{A}} \times \Delta^{\text {op }}}$ have Reedy model category structures, with weak equivalences and cofibrations defined at each simplicial space W. $\{\mathbf{B}\}$ for each $\mathbf{B} \in \mathbf{\Theta}^{\mathcal{A}}$. 
As in 2.12 there is also a resolution model category structure on the category $\left(\mathcal{S}_{*}^{\Theta^{\mathcal{A}}}\right)^{\Delta^{\mathrm{op}}}=\mathcal{S}_{*}^{\Theta^{\mathcal{A}} \times \Delta^{\mathrm{op}}}$ of simplicial dual $\mathcal{A}$-presheaves. Again, if a simplicial presheaf $\mathfrak{W}_{\bullet}$ is cofibrant, each $\mathfrak{W}_{n}$ is weakly equivalent to a coproduct of free dual strict $\mathcal{A}$-mapping algebras, so it is a dual weak $\mathcal{A}$-mapping algebra, and $\mathfrak{W}_{\bullet} \rightarrow \mathfrak{X}$ is a resolution of dual weak $\mathcal{A}$-mapping algebras only if $\pi_{0} \mathfrak{W} \bullet \rightarrow \pi_{0} \mathfrak{X}$ is a resolution of $\Pi^{\mathcal{A}}$-algebras.

Since $\mathcal{S}_{[n]}$ is still proper, we also have a resolution model category structure on the category $\mathcal{S}_{[n]}^{\Theta^{\mathcal{A}} \times \Delta^{\text {op }}}$ of $n$-truncated simplicial dual $\mathcal{A}$-presheaves $(\$ 1.23)$.

The Eckmann-Hilton dual of Theorem 2.13 has the following more involved form:

2.22. Theorem. Let $R$ be any commutative ring, $\mathcal{C}=\mathcal{S}_{*}$, and $\mathfrak{X}$ a dual strict $R$ mapping algebra (for $\Theta^{R}=s \mathcal{M}_{\lambda}^{R}$ as in $\lcm{2.15}$ ), which we assume to be a dual strict Stover mapping algebra.

(a) There is a functor associating to $\mathfrak{X}$ a cosimplicial object $\mathbf{W}^{\bullet} \in \mathcal{S}_{*}^{\Delta}$ with each $W^{n}$ in $s \mathcal{M}_{\lambda}^{R}$, equipped with a natural augmentation of $R$-mapping algebras $\varepsilon: \mathfrak{M}^{R} \mathbf{W}^{\bullet} \rightarrow \mathfrak{X}$, such that $\pi_{0} \mathfrak{M}^{R} \mathbf{W}^{\bullet} \rightarrow \pi_{0} \mathfrak{X}\{M\} \quad$ is a simplicial resolution of $\Pi^{\mathcal{A}}$-algebras.

(b) If $\mathfrak{X} \in \mathrm{sMap}_{\mathrm{re}}^{\mathrm{St}, R}$ is weakly equivalent to $\mathfrak{M}^{\mathrm{St}, R} \mathbf{Y} \quad$ (for some $R$-good space $\mathbf{Y}$ ), then $\operatorname{Tot} \mathbf{W}^{\bullet}$ is homotopy equivalent to the $R$-completion of $\mathbf{Y}$ (so in particular Tot $\mathbf{W}^{\bullet}$ realizes $\mathfrak{X}$ up to weak equivalence).

(c) When $R$ is a field, we can start with any dual strict $\mathcal{A}$-mapping algebra $\hat{\mathfrak{X}}$ (for $\mathcal{A}=\{K(R, n)\}_{n=1}^{\infty} \quad$ in $(1.14)$. If it extends to a dual strict Stover mapping algebra $\mathfrak{X}$ as defined in 2.16 , and then (a) and (b) hold.

(d) When $R=\mathbb{F}_{p}$ or $\mathbb{Q}$, and $\mathfrak{X}$ is simply connected (that is, letting $\mathcal{A}=$ $\{K(R, n)\}_{n=2}^{\infty}$ in $\sqrt{1.14}$ ), any $R$-mapping algebra (for a suitable limit cardinal $\lambda$ ) is weakly equivalent to $\mathfrak{M}^{\mathrm{St}, R} \mathbf{Y}$ for some simply connected $\mathbf{Y}$, unique up to R-equivalence.

Proof. This follows from various results in BS2]:

(a) This is [BS2, Proposition 3.9].

(b) This is [BS2, Theorem 3.26].

(c) This combines [BS2, Proposition 2.23] and [BS2, Theorem 2.30], using the fact that a weak equivalence of dual strict Stover mapping algebras $\mathfrak{f}: \mathfrak{X} \rightarrow \mathfrak{Y}$ induces weak equivalence (in the model category of [Bou2, §3]) between the corresponding cosimplicial spaces (see [Bou2, $\S 7.7]$ ).

(d) This is [BS2, Theorem 4.23] (when $\lambda=\aleph_{0}$ ) or [BS2, Theorem 4.28] (otherwise).

2.23. Corollary. If $R$ is any commutative ring, there is a realization functor $N$ : $\left(\mathrm{sMap}_{\mathrm{re}}^{\mathrm{St}, R}\right)^{\mathrm{op}} \rightarrow \mathcal{S}_{*} \quad$ with a natural weak equivalence $\varepsilon: \mathrm{Id} \rightarrow N \circ \mathfrak{M}^{\mathrm{St}, R}$. Thus any functor $\mathbf{T}: \mathcal{S}_{R} \rightarrow \mathcal{D}$ (see (2.20) to a model category $\mathcal{D}$ which preserves $R$ equivalences induces a functor $\mathfrak{T}:=\mathbf{T} \circ N:\left(\mathrm{sMap}_{\mathrm{re}}^{\mathrm{St}, R}\right)^{\mathrm{op}} \rightarrow \mathcal{D}$ equipped with a natural weak equivalence $\vartheta=\mathbf{T} \varepsilon: \mathfrak{T} \rightarrow \mathfrak{T} \circ \mathfrak{M}^{\mathrm{St}, R}$.

Proof. We set $N:=$ Tot $\mathbf{W}^{\bullet}$, where $\mathfrak{X} \mapsto \mathbf{W}^{\bullet} \quad$ is the functor of Theorem 2.22 Once we know that $\mathfrak{X}$ is weakly $R$-good (see $\ 2.20$ ), the natural augmentation $\varepsilon$ : $\mathfrak{M}^{\mathrm{St}, R} N \rightarrow \mathfrak{X} \quad$ is a weak equivalence by Theorem 2.22 (b) or (c). 
2.24. Example. For any $\mathbf{Z} \in \mathcal{S}_{*}$ with $\mathbf{T}: \mathcal{S}_{R} \rightarrow \mathcal{S}_{*}$ the functor $\operatorname{map}_{*}(\mathbf{Z},-)$, the induced functor $\mathfrak{T}:=\mathbf{T} \circ N: \mathrm{sMap}_{\mathrm{re}}^{\mathrm{St}, R} \rightarrow \mathcal{S}_{*}$ has the property that if $\mathfrak{Z}:=\mathfrak{M}^{\mathrm{St}, R} \mathbf{Z}$ and $\mathfrak{X}$ is the realizable dual strict Stover mapping algebra $\mathfrak{M}^{\mathrm{St}, R} \mathbf{Y}$ for some $R$-good space $\mathbf{Y}$, then $\mathfrak{T}(\mathfrak{X})$ is weakly equivalent to $\mathfrak{Z}\{\mathbf{Y}\}$.

Thus the $n$-truncation $P^{n} \mathfrak{T} \quad$ (cf. 11.23 ) when evaluated at $\mathfrak{X}=\mathfrak{M}^{\mathrm{St}, R} \mathbf{Y}$, is determined by $P^{n} \mathfrak{Z}$. Moreover, from the alternative description in $\$ 1.9$ we see that if $\mathbf{Y} \in \Theta_{\lambda}^{\mathrm{St}, R}$, then $\mathfrak{T}(\mathfrak{X})$ corresponds to the $n$-truncated simplicial category $P^{n} \mathcal{X}$, so that in fact $P^{n} \mathfrak{T}$, when evaluated at free dual strict Stover mapping algebras, factors through the $n$-truncation.

\section{RELATIVE DERIVED FUnCTORS}

Let $\mathbf{T}: \mathcal{C} \rightarrow \mathcal{D}$ be a homotopy functor between model categories of spaces. We want to study the homotopy spectral sequence for the (co)simplicial object obtained by applying $\mathbf{T}$ to a (co)simplicial resolution of a space $\mathbf{Y} \in \mathcal{C}$, using a relative version of the total derived functor of the associated functor of mapping algebras $\mathfrak{T}$.

3.1. Relative left and right derived functors. If $T: \mathcal{D} \rightarrow \mathcal{E}$ is a functor between model categories which preserves weak equivalences of cofibrant objects, recall that Quillen constructs the total left derived functor $\mathbf{L} T:$ ho $\mathcal{D} \rightarrow$ ho $\mathcal{E}$ on an object $x \in \mathcal{D}$ by applying $T$ to any cofibrant replacement of $x$ (see [Q2, I, §4]). In order for this to work, $T$ need only be defined on the full subcategory $\mathcal{D}_{\text {cof }}$ of all cofibrant objects in $\mathcal{D}$. In the spirit of the Eilenberg-Moore "relative homological algebra" (see [EM]), one could require only that $T$ be defined on some full subcategory $\mathcal{P}$ of special cofibrant objects in $\mathcal{D}_{\text {cof }}$ (e.g., free, rather than projective, resolutions) - as long as every object of $\mathcal{D}$ is weakly equivalent to an object of $\mathcal{P}$ (and $T$ still takes weakly equivalent objects of $\mathcal{P}$ to weakly equivalent objects in $\mathcal{D}$ ). Moreover, if are only given a full subcategory $\mathcal{D}_{\mathcal{P}}$ of $\mathcal{D}$, closed under weak equivalences, and every object of $\mathcal{D}_{\mathcal{P}}$ is weakly equivalent to one in $\mathcal{P}$, we still have $\mathbf{L} T:$ ho $\mathcal{D}_{\mathcal{P}} \rightarrow$ ho $\mathcal{E}$. Finally, $\mathcal{E}$ need not be a model category - all we need is the localization $\gamma: \mathcal{E} \rightarrow$ ho $\mathcal{E}$, with $\gamma \circ T$ taking weak equivalences to isomorphisms.

However, we shall be interested in a situation where we have two model category structures on $\mathcal{D}$ - or perhaps only a subcategory $\mathcal{W}^{\prime}$ of the given weak equivalences $\mathcal{W}$. This commonly occurs when our model category $\left(\mathcal{D}, \mathcal{W}, \mathcal{D}_{\text {cof }}, \mathcal{D}_{\text {fib }}\right)$ is obtained by localizing another.

In this case, we shall assume that $\mathcal{P}$ and $\mathcal{D}_{\mathcal{P}}$ satisfy the stronger requirement that for each $x \in \mathcal{D}_{\text {cof }} \cap \mathcal{D}_{\mathcal{P}}$ there is a map $f: y \rightarrow x$ in $\mathcal{W}^{\prime}$ with $y \in \mathcal{P}$. If $T: \mathcal{P} \rightarrow \mathcal{E}$ is then a functor which preserves $\mathcal{W}$-weak equivalences, the relative left derived functor of $T$ (with respect to $\mathcal{P}$ and $\mathcal{W}^{\prime}$ ) is the functor $\mathbf{L}^{\text {rel }} T:$ ho $\mathcal{D} \rightarrow$ ho $\mathcal{E}$ defined on $z \in \mathcal{D}_{\mathcal{P}}$ by applying $T$ to $y$, where $g: x \rightarrow z$ is a cofibrant replacement (with respect to $\mathcal{W}$ ) and $f: y \rightarrow x$ in $\mathcal{W}^{\prime}$ is as above.

Dually, if we have full subcategories $\mathcal{F}$ of $\mathcal{D}_{\text {fib }}$ (the fibrant objects) and $\mathcal{D}_{\mathcal{F}}$ of $\mathcal{D}$, both closed under weak equivalences, and $\mathcal{W}^{\prime} \subseteq \mathcal{W}$, with the corresponding dual properties with respect to a homotopy functor $T: \mathcal{F} \rightarrow \mathcal{E}$, the relative right derived

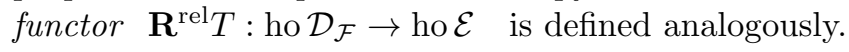

3.2. Remark. In the applications we have in mind, $\mathcal{D}$ will be a resolution model category of simplicial mapping algebras, so the weak equivalences $\mathcal{W}$ in $\mathcal{D}$ are $E^{2}$ equivalences. However, we also have a Reedy model structure on $\mathcal{D}$, and the special 
weak equivalences $\mathcal{W}^{\prime}$ will be the $E^{1}$-equivalences. The ability to apply the functor $T$ to a resolution which is $\mathcal{W}^{\prime}$-equivalent to any cofibrant replacement (that is, simplicial resolution) $y$ of an object $z \in \mathcal{D}_{\mathcal{F}}$ provides the flexibility we want in using particular resolutions - e.g., minimal - to calculate $\left(\mathbf{L}^{\text {rel }} T\right) z$, and eventually, the appropriate terms of the spectral sequence.

\section{A. Relative left derived functors of mapping algebras}

For $\mathcal{C}=\mathcal{G}$ and $\boldsymbol{\Theta}_{\mathcal{B}}$ as in $₫ 1.4$, let $\mathbf{W} \bullet$ be a resolution of $\mathbf{Y} \in \mathcal{G}$ in the resolution model category structure on $\mathcal{G}^{\Delta^{\mathrm{op}}}$. Given a homotopy functor $\mathbf{T}: \mathcal{G} \rightarrow \mathcal{D}$ for $\mathcal{D}$ a "category of spaces" such as $\operatorname{Top}_{0}, \mathcal{S}_{*}$, or $\mathcal{G}$, we wish to study the homotopy spectral sequence for the simplicial space $\mathbf{T W} \bullet \in \mathcal{D}^{\Delta^{\mathrm{op}}}$

By applying the functor $\mathfrak{M}_{\mathrm{St}}: \mathcal{C} \rightarrow \operatorname{sMap}_{\mathrm{St}}$ of 2.16 to $\mathbf{W}_{\bullet}$, we obtain a simplicial strict Stover mapping algebra $\mathfrak{W}_{\bullet}:=\mathfrak{M}_{\mathrm{St}} \mathbf{W}_{\bullet} \quad$ which is a cofibrant replacement for $\mathfrak{X}:=\mathfrak{M}_{\mathrm{St}} \mathbf{Y}$ in the resolution model category structure on $\mathcal{S}_{*}^{\Theta_{\mathcal{B}}^{\mathrm{op}} \times \Delta^{\mathrm{op}}}$ associated to the free dual strict Stover mapping algebras $\left\{\mathfrak{M}_{\mathrm{St}} \mathbf{S}^{i}\right\}_{i=1}^{\infty}$. By Corollary 2.14, there is functor $\mathfrak{T}=\mathbf{T} N: \operatorname{sMap}_{\mathrm{St}} \rightarrow \mathcal{D}$, with a natural Reedy (that is, levelwise) weak equivalence of simplicial spaces $\vartheta: \mathfrak{T W}_{\bullet} \rightarrow \mathbf{T W}_{\bullet}$.

We want to calculate the total left derived functor of $\mathfrak{T}$ evaluated at $\mathfrak{X}$ by applying $\mathfrak{T}$ to any resolution $\mathfrak{V}_{\bullet} \rightarrow \mathfrak{X}$. However, such an $\mathfrak{V}_{\bullet}$ is just a simplicial $\mathcal{B}$-presheaf, and the functor $\mathfrak{T}$ is only defined for strict Stover mapping algebras. As explained in 3.1 . our solution to this difficulty is to show that any such $\mathfrak{V} \bullet$ is in fact $E^{1}$ equivalent to a simplicial strict $\mathcal{B}$-mapping algebra $\mathfrak{W}_{\bullet}$. For this purpose we require some additional notions from [BJT2, §1]:

3.3. $\mathbf{C W}$ resolutions. If $\mathcal{E}$ is any pointed complete category, the $n$-th Moore chains object of $G_{\bullet} \in \mathcal{E}^{\Delta^{\mathrm{op}}}$ is $C_{n} G_{\bullet}:=\cap_{i=1}^{n} \operatorname{Ker}\left\{d_{i}: G_{n} \rightarrow G_{n-1}\right\}$. The differential is $\partial_{n}:=\left.d_{0}\right|_{\mathcal{C}_{n} G_{\bullet}}: C_{n} G_{\bullet} \rightarrow C_{n-1} G_{\bullet}$ and the cycles objects is $Z_{n} G_{\bullet}:=\operatorname{Ker}\left(\partial_{n}\right)$, with $v_{n}: Z_{n} G_{\bullet} \rightarrow C_{n} G_{\bullet} \quad$ the inclusion. These are defined for any restricted simplicial object $G_{\bullet} \in \mathcal{E}^{\Delta_{+}^{\mathrm{op}}} \quad($ see 0.4$)$.

The $n$-th latching object for $G_{\bullet}$ is the colimit

$$
L_{n} G_{\bullet}:=\operatorname{colim}_{\theta^{\mathrm{op}}:[\mathbf{k}] \rightarrow[\mathbf{n}]} G_{k},
$$

where $\theta$ ranges over the surjective maps $[\mathbf{n}] \rightarrow[\mathbf{k}]$ in $\Delta$ for $k<n$.

A simplicial object $G_{\bullet} \in \mathcal{E}^{\Delta^{\mathrm{op}}}$ is called a $C W$ object if it is equipped with a $C W$ basis $\left(\bar{G}_{n}\right)_{n=0}^{\infty}$ in $\mathcal{E}$ such that $G_{n}=\bar{G}_{n} \amalg L_{n} G_{\bullet}$, and $\left.d_{i}\right|_{\bar{G}_{n}}=0$ for $1 \leq i \leq n$. The $n$-th attaching map for $G_{\bullet} \quad$ is defined to be $\partial_{n}^{G}:=\left.d_{0}\right|_{G_{n}}: \bar{G}_{n} \rightarrow C_{n-1} G_{\bullet}$ (which actually lands in $Z_{n-1} G_{\bullet}$ ).

When $\mathcal{E}$ is a suitable category of universal algebras, such as $\Pi_{\mathcal{B}}-\mathrm{Alg} \quad$ (cf. 11.11), a simplicial object $V_{\bullet} \in \mathcal{E}^{\Delta^{\text {op }}}$ with an augmentation to $\Lambda \in \mathcal{C}$ is called a $C W$ resolution if $V_{\bullet} \rightarrow \Lambda$ is acyclic, with a CW basis $\left(\bar{V}_{n}\right)_{n=0}^{\infty}$ having each $\bar{V}_{n}$ free. Moreover, in this case $\partial_{n}^{V}$ surjects onto $Z_{n-1} V_{\bullet} \quad\left(\right.$ where $Z_{-1} V_{\bullet}:=\Lambda$ ).

For $\mathcal{B}=\left\{\mathbf{S}^{i}\right\}_{i=1}^{\infty}$, by BJT2, Lemma 1.38] every free simplicial $\Pi_{\mathcal{B}}$-algebra (1.11) has a free CW basis. Moreover, by [BJT2, Theorem 2.29], every CW resolution $V_{\bullet}$ of a realizable $\Pi_{\mathcal{B}}$-algebra $\Lambda=\pi_{*} \mathbf{Y}=\pi_{0} \mathfrak{M}_{\mathcal{B}} \mathbf{Y} \quad$ can be realized by an augmented simplicial space $\mathbf{W}_{\bullet} \rightarrow \mathbf{Y}$. Therefore, every free simplicial $\Pi_{\mathcal{B}}$-algebra resolution $V_{\bullet} \rightarrow \pi_{*} \mathbf{Y}$ can be realized (non-canonically) by a simplicial resolution of strict 
$\mathcal{B}$-mapping algebras $\mathfrak{W}_{\bullet} \rightarrow \mathfrak{M}_{\mathcal{B}} \mathbf{Y}$, with $\pi_{0} \mathfrak{W}_{\bullet} \cong V_{\bullet}$. In order to apply the ideas of 3.1 , we must show that any simplicial $\mathcal{B}$-presheaf resolution $\mathfrak{V}_{\bullet}$ of $\mathfrak{X}=\mathfrak{M}_{\mathcal{B}} \mathbf{Y}$ is Reedy weakly equivalent to a strict Stover mapping algebra resolution $\mathfrak{W}_{\bullet}$. To do so, we recall the following constructions from [BJT2]:

3.5. Sequential realizations. Assume given an enriched sketch $\boldsymbol{\Theta}_{\mathcal{B}}=\boldsymbol{\Theta}_{(\mathcal{B}, \mathcal{F}, \mathcal{E})}$ in a pointed simplicial model category $\mathcal{C}$, as in 1.1 and a $\mathrm{CW}$-resolution $V_{\bullet}$ of a

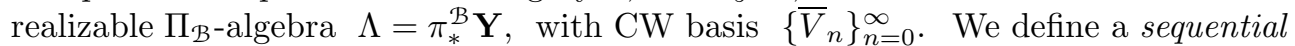
realization of $V_{\bullet} \quad($ for $\mathbf{Y})$ to be a sequence $\mathcal{W}$ of maps

$$
\mathbf{W}_{\bullet}^{[0]} \stackrel{\iota^{[0]}}{\longrightarrow} \mathbf{W}_{\bullet}^{[1]} \stackrel{\iota^{[1]}}{\longrightarrow} \mathbf{W}_{\bullet}^{[2]} \rightarrow \ldots \mathbf{W}_{\bullet}^{[n]} \stackrel{\iota^{[n]}}{\longrightarrow} \mathbf{W}_{\bullet}^{[n+1]} \rightarrow \ldots
$$

between Reedy fibrant and cofibrant objects in $C^{\Delta^{\text {op }}}$, such that for each $n \geq 0$ :

(i) $\quad \overline{\mathbf{W}}_{n} \in \boldsymbol{\Theta}_{\mathcal{B}} \quad$ realizes the given $\mathrm{CW}$ basis $\Pi^{\mathcal{A}}$-algebra $\bar{V}_{n}$.

(ii) There is an $n$-skeletal restricted simplicial object $\widetilde{\mathbf{W}}_{\bullet}^{[n]}$ with

$$
\widetilde{\mathbf{W}}_{k}^{[n]}=\mathbf{W}_{k}^{[n-1]} \amalg C \Sigma^{n-k-1} \overline{\mathbf{W}}_{n} \quad \text { for } 0 \leq k \leq n,
$$

where by convention $C \Sigma^{0} \overline{\mathbf{W}}_{n}:=C \overline{\mathbf{W}}_{n}, \quad C \Sigma^{-1} \overline{\mathbf{W}}_{n}:=\overline{\mathbf{W}}_{n}$. and $\mathbf{W}_{\bullet}^{[-1]}=$ *.

(iii) The face map $\left.d_{0}\right|_{C \Sigma^{n-k-1} \overline{\mathbf{W}}_{n}}$ is the map $F_{k}$ in the commuting diagram

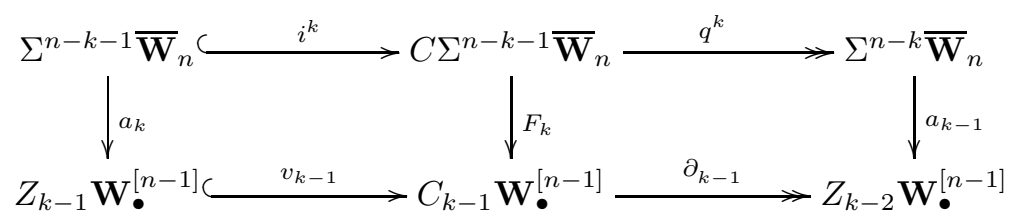

in which the top row is a strict cofibration sequence and the bottom row a strict fibration sequence in $\mathcal{C}$. Thus $F_{k}$ is a nullhomotopy for $v_{k-1} \circ a_{k}$, which in turn defines $a_{k-1}$, using (3.8). The first face map $\left.d_{1}\right|_{C \Sigma^{n-k-1}} \overline{\mathbf{W}}_{n}$ is the composite $C \Sigma^{n-k-1} \overline{\mathbf{W}}_{n} \stackrel{q^{k}}{\longrightarrow} \Sigma^{n-k} \overline{\mathbf{W}}_{n} \stackrel{i^{k-1}}{\longrightarrow} C \Sigma^{n-k} \overline{\mathbf{W}}_{n}$, and $\left.d_{i}\right|_{C \Sigma^{n-k-1}} \overline{\mathbf{W}}_{n}=$ 0 for $i>1$.

We start with $F_{n}: \overline{\mathbf{W}}_{n} \rightarrow C_{n-1} \mathbf{W}_{\bullet}^{[n-1]}$ a realization of the $n$-th attaching map $\partial_{n}^{V}: \bar{V}_{n} \rightarrow C_{n-1} V_{\bullet}$ for the given $\mathrm{CW}$ resolution, and $a_{n-1}:=$ $\partial_{n-1} \circ F_{n}: \overline{\mathbf{W}}_{n} \rightarrow Z_{n-2} \mathbf{W}_{\bullet}^{[n-1]} \quad$ (with $v_{n-2} \circ a_{n-1}$ indeed nullhomotopic).

(iv) Let $\widehat{\mathbf{W}}_{\bullet}^{[n]}$ be the pushout of the obvious maps

$$
\mathbf{W}_{\bullet}^{[n-1]} \leftarrow \mathcal{L} i^{*} \mathbf{W}_{\bullet}^{[n-1]} \rightarrow \mathcal{L} \widetilde{\mathbf{W}}_{\bullet}^{[n]}
$$

where $\mathcal{L}: \mathcal{C}^{\Delta_{+}^{\mathrm{op}}} \rightarrow \mathcal{C}^{\Delta^{\mathrm{op}}}$ is the left adjoint of $i^{*}: \mathcal{C}^{\Delta^{\mathrm{op}}} \rightarrow \mathcal{C}^{\Delta_{+}^{\mathrm{op}}}$, as in 0.4 We then let $\mathbf{W}_{\bullet}^{[n]}$ be a Reedy fibrant and cofibrant replacement for $\widehat{\mathbf{W}}_{\bullet}^{[n]}$.

(v) There is an augmentation $\varepsilon^{[n]}: \mathbf{W}_{\bullet}^{[n]} \rightarrow \mathbf{Y} \quad$ realizing $V_{\bullet} \rightarrow \Lambda$ through simplicial dimension $n$ - that is, the $n$-truncation of the augmented simplicial $\Pi^{\mathcal{A}}$-algebra $\pi_{*}^{\mathcal{A}} \mathbf{W}_{\bullet}^{[n]} \rightarrow \pi_{*}^{\mathcal{A}} \mathbf{Y} \quad$ is isomorphic to the $n$-truncation of $V_{\bullet} \rightarrow \Lambda$.

(vi) The maps $\iota^{[n]}$ restrict to a trivial cofibration $\iota_{k}^{[n]}: \mathbf{W}_{k}^{[n-1]} \stackrel{\simeq}{\longrightarrow} \mathbf{W}_{k}^{[n]}$ for each $0 \leq k<n$.

It follows that $\mathbf{W}_{\bullet}:=\operatorname{colim}_{n} \mathbf{W}_{\bullet}^{[n]} \stackrel{\varepsilon}{\rightarrow} \mathbf{Y}$ is a simplicial resolution in the resolution model category $\mathcal{C}^{\Delta^{\mathrm{op}}}$. See [BJT2, §2] for further details. 
3.10. Theorem. For an enriched sketch $\boldsymbol{\Theta}_{\mathcal{B}}$ as in 1 1.1, $\mathbf{Y} \in \mathcal{C}$ fibrant, and $\mathfrak{X}:=\mathfrak{M}_{\mathcal{B}} \mathbf{Y}$, let $\boldsymbol{\eta}: \mathfrak{V}_{\bullet} \rightarrow c(\mathfrak{X})$. be a trivial fibration with $\mathfrak{V}_{\bullet}$ cofibrant in $\mathcal{S}_{*}^{\Theta_{\mathcal{B}}^{\mathrm{op}} \times \Delta^{\mathrm{op}}}$. Then for any sequential realization $\mathcal{W}$ of the $\Pi_{\mathcal{B}}$-algebra resolution $\pi_{0} \mathfrak{V}_{\bullet} \rightarrow \pi_{*}^{\mathcal{B}} \mathbf{Y} \quad$ as in 3 3.5, there is a Reedy weak equivalence of simplicial weak $\mathcal{B}$-mapping algebras $\mathfrak{f}: \mathfrak{M}_{\mathcal{B}} \mathbf{W}_{\bullet} \rightarrow \mathfrak{V}_{\bullet}$.

Proof. By 2.12, the simplicial $\Pi_{\mathcal{B}}$-algebra $V_{\bullet}:=\pi_{o} \mathfrak{V}_{\bullet}$ is a free resolution of the

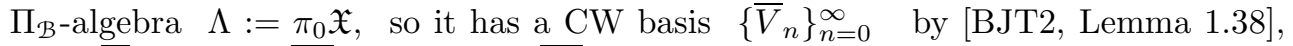
with $\bar{V}_{n}=\pi_{0} \mathfrak{M}_{\mathcal{B}} \overline{\mathbf{W}}_{n}$ for some $\overline{\mathbf{W}}_{n} \in \operatorname{Obj} \boldsymbol{\Theta}_{\mathcal{B}}$. We may assume $\boldsymbol{\Theta}_{\mathcal{B}}$ contains all simplicial groups of the homotopy type of a (possibly trivial) wedge of objects of $\mathcal{B}$ of cardinality $<\lambda$. This will ensure that all objects $\mathbf{W}_{k}^{[n]}, \quad \widetilde{\mathbf{W}}_{k}^{[n]}, \quad \widehat{\mathbf{W}}_{k}^{[n]}$, and so on, in 3.5 are in $\boldsymbol{\Theta}_{\mathcal{B}}$.

We construct $\mathfrak{f}$ by a double induction: in the outer induction, we construct maps of simplicial weak $\mathcal{B}$-mapping algebras $\mathfrak{f}^{[n]}: \mathfrak{M}_{\mathcal{B}} \mathbf{W}_{\bullet}^{[n]} \rightarrow \mathfrak{V}_{\bullet}$. Assuming we have defined $\mathfrak{f}^{[n-1]}$, we need to extend it to a map of $n$-truncated restricted simplicial objects $\widetilde{\mathfrak{f}}^{[n]}: \mathfrak{M}_{\mathcal{B}} \widetilde{\mathbf{W}}_{\bullet}^{[n]} \rightarrow \mathfrak{V}_{\bullet}$, which we do by an inner downward induction on $0 \leq k \leq n$. Using Lemma 1.8, we see from (3.7) that $\widetilde{\mathfrak{f}}_{k}^{n]}$ is determined by an element $\bar{f}_{n}^{(k)} \in \mathfrak{V}_{k}\left\{C \Sigma^{n-k-1} \overline{\mathbf{W}}_{n}\right\}_{0}$ with $d_{i} \bar{f}_{n}^{(k)}=0$ for $i \geq 2$.

Step A. To start the outer induction, note that since $\mathbf{W}_{0}^{[0]}=\overline{\mathbf{W}}_{0}$, by Lemma 1.8 the augmentation $\varepsilon^{[0]}: \mathfrak{M}_{\mathcal{B}} \mathbf{W}_{\bullet}^{[0]} \rightarrow \mathfrak{X} \quad$ is determined by an element $e \in$ $\mathfrak{X}\left\{\overline{\mathbf{W}}_{0}\right\}_{0}=\operatorname{Hom}\left(\overline{\mathbf{W}}_{0}, \mathbf{Y}\right)$. Since $\boldsymbol{\eta}: \mathfrak{V}_{\bullet} \rightarrow c(\mathfrak{X})$. is a Reedy fibration (see [J] $\S 2]), \quad\left(\boldsymbol{\eta}_{0}\right)_{*}: \mathfrak{V}_{0}\left\{\overline{\mathbf{W}}_{0}\right\} \rightarrow \mathfrak{X}\left\{\overline{\mathbf{W}}_{0}\right\} \quad$ is a fibration and in particular a surjection in $\mathcal{S}_{*}$. Moreover, $\pi_{0} \mathfrak{V}_{0} \cong \pi_{*}^{\mathcal{B}} \overline{\mathbf{W}}_{0}$ is a free $\Pi_{\mathcal{B}}$-algebra, by our assumption on $\mathfrak{V}_{\bullet}$, so we have an element $\bar{f}_{0}^{(0)} \in \mathfrak{V}_{0}\left\{\overline{\mathbf{W}}_{0}\right\}_{0} \quad$ representing Id $\in \pi_{0} \mathfrak{V}_{0}\left\{\overline{\mathbf{W}}_{0}\right\} \quad$ with $\left(\boldsymbol{\eta}_{0}\right)_{*} \bar{f}_{0}^{(0)}=e$ by [BJT1, Lemma 15.9], as required.

Step B. Given $\mathfrak{f}^{[n-1]}: \mathfrak{M}_{\mathcal{B}} \mathbf{W}_{\bullet}^{[n-1]} \rightarrow \mathfrak{V}_{\bullet}$, consider the augmented simplicial space $\mathbf{X}_{\bullet}:=\mathfrak{V}_{\bullet}\left\{\overline{\mathbf{W}}_{n}\right\} \rightarrow \mathfrak{X}\left\{\overline{\mathbf{W}}_{n}\right\}:$ we think of this as a bisimplicial set with vertical direction internal to each $\mathfrak{V}_{k}\left\{\overline{\mathbf{W}}_{n}\right\} \in \mathcal{S}_{*}$, and horizontal direction corresponding to the original simplicial direction of $\mathfrak{V}_{\bullet}$. The (split) inclusion $j_{n}: \bar{V}_{n} \hookrightarrow V_{n}$ for the CW basis $\Pi_{\mathcal{B}}$-algebra $\bar{V}_{n}=\pi_{0} \mathfrak{M}_{\mathcal{B}} \overline{\mathbf{W}}_{n}$ corresponds by the $\Pi_{\mathcal{B}}$-algebra analogue of Lemma 1.8 (the ordinary Yoneda embedding) to an element $\tilde{\jmath}_{n} \in V_{n}\left\{\overline{\mathbf{W}}_{n}\right\}$ - that is. a homotopy class $\left[\bar{f}_{n}^{(n)}\right] \in \pi_{0} \mathbf{X}_{n}=\pi_{0} \mathfrak{V}_{n}\left\{\overline{\mathbf{W}}_{n}\right\}$. Since $\mathbf{Y}$ is fibrant in $\mathcal{C}, \mathfrak{X}=\mathfrak{M}_{\mathcal{B}} \mathbf{Y}$ is fibrant in $\mathcal{S}_{*}^{\boldsymbol{\Theta}^{\mathcal{A}}}$, so $c(\mathfrak{X}) \bullet$ is Reedy fibrant. But $\mathfrak{V}_{\bullet} \rightarrow c(\mathfrak{X}) \bullet$ is a Reedy fibration, so $\mathfrak{V}_{\bullet}$ is Reedy fibrant, and therefore $\mathbf{X}_{\bullet}$ is, too. Thus by [Sto, Lemma 2.7] the inclusion of the (horizontal) Moore object $C_{n} \mathbf{X}_{\bullet}:=C_{n}^{h} \mathbf{X} \bullet \hookrightarrow \mathbf{X}_{n}$ induces an isomorphism $\pi_{0} C_{n} \mathbf{X} \bullet \rightarrow C_{n} \pi_{0} \mathbf{X} \bullet=C_{n} V_{\bullet}\left\{\overline{\mathbf{W}}_{n}\right\} \quad$ (see also [BJT2, Lemma 1.30]).

The functor $\mathfrak{M}_{\mathcal{B}}$ of 1.7 takes any pointed limit in $\mathcal{C}$ to the corresponding limit of $\mathcal{B}$-presheaves, so $C_{n-1} \mathfrak{M}_{\mathcal{B}} \mathbf{W}_{\bullet}^{[n-1]}=\mathfrak{M}_{\mathcal{B}} C_{n-1} \mathbf{W}_{\bullet}^{[n-1]}$, and thus the attaching map $d_{0}=\partial_{n}^{W}: \overline{\mathbf{W}}_{n} \rightarrow C_{n-1} \mathbf{W}_{\bullet}^{[n-1]} \quad$ corresponds under Lemma 1.8 to an element $\gamma \in$ $C_{n-1} \mathfrak{M}_{\mathcal{B}} \mathbf{W}_{\bullet}^{[n-1]}\left\{\overline{\mathbf{W}}_{n}\right\}$. Moreover, the given map of $\mathcal{B}$-presheaves $\mathfrak{f}^{[n-1]}$ induces $C_{n-1} \mathfrak{f}^{[n-1]}: C_{n-1} \mathfrak{M}_{\mathcal{B}} \mathbf{W}^{[n-1]} \rightarrow C_{n-1} \mathfrak{V}_{\bullet}$, which takes $\gamma$ to an element $\psi_{n}:=$ $\mathfrak{f}^{[n-1]}(\gamma) \in\left(C_{n-1}^{h} \mathbf{X}_{\bullet}\right)_{0}$. 
Since $\mathbf{X}_{\bullet} \quad$ is Reedy fibrant, the matching structure map $\delta_{n}: \mathbf{X}_{n} \rightarrow M_{n} \mathbf{X} \bullet \quad$ is a fibration (cf. [H, §16.3]), and we have an inclusion $\iota: C_{n-1} \mathbf{X}_{\bullet} \hookrightarrow M_{n} \mathbf{X}_{\bullet}$, given by $x \mapsto(x, x, 0, \ldots, 0)$. Because $\mathbf{W}_{\bullet}$ realizes the $\mathrm{CW}$ resolution $V_{\bullet} \rightarrow \Lambda$ of $\Pi^{\mathcal{A}}-$ algebras and $j_{n}: \bar{V}_{n} \hookrightarrow V_{n}$ factors through $C_{n} V_{\bullet}$, we have $\left(\delta_{n}\right)_{*}\left[\bar{f}_{n}^{(n)}\right]=\left[\iota \circ \psi_{n}\right]$. We may therefore change $\bar{f}_{n}^{(n)}$ within its homotopy class so that $\delta_{n}\left(\bar{f}_{n}^{(n)}\right)=\iota \circ \psi$ on the nose.

Lemma [1.8 together with (3.7) (and our assumption that $\mathbf{W}_{k}^{[n-1]}$ and $C \Sigma^{n-k-1} \overline{\mathbf{W}}_{n}$ are in $\left.\boldsymbol{\Theta}_{\mathcal{B}}\right)$ imply that $\mathfrak{M}_{\mathcal{B}} \widetilde{\mathbf{W}}_{n}^{[n]}$ is the coproduct of $\mathfrak{M}_{\mathcal{B}} \mathbf{W}_{n}^{[n-1]}$ and $\mathfrak{M}_{\mathcal{B}} \overline{\mathbf{W}}_{n}$. Therefore, this choice of $\bar{f}_{n}^{(n)}$ defines a map of $\mathcal{B}$-presheaves $\mathfrak{f}^{[n]}: \mathfrak{M}_{\mathcal{B}} \widetilde{\mathbf{W}}_{n}^{[n]} \rightarrow \mathfrak{V}_{n} \quad$ (extending $\mathfrak{f}^{[n-1]}$ ). Since $\left.F_{n-1}\right|_{\overline{\mathbf{W}}_{n}}=d_{0}^{h}(\gamma) \quad$ (in the notation of $₫ 3.5\left(\right.$ iii)), we have $d_{0}^{h} \bar{f}_{n}^{(n)}=d_{1}^{h} \bar{f}_{n}^{(n)}$.

Step C. In the $k$-th stage of the inner (downward) induction, with $k<n$, we assume that for each for $k<j \leq n$ we have chosen a map of weak $\mathcal{B}$-mapping algebras $\bar{f}_{j}^{(n)}: \mathfrak{M}_{\mathcal{B}} C \Sigma^{n-j-1} \overline{\mathbf{W}}_{n} \rightarrow \mathfrak{V}_{j}$, represented by an element $\psi_{j} \in \mathfrak{V}_{j}\left\{C \Sigma^{n-j-1} \overline{\mathbf{W}}_{n}\right\}_{0}$ with $d_{i}^{h} \psi_{j}=0$ for $2 \leq i \leq j$. If $\iota_{n-j-1}: \Sigma^{n-j-1} \overline{\mathbf{W}}_{n} \hookrightarrow C \Sigma^{n-j-1} \overline{\mathbf{W}}_{n}$ is the inclusion, then $\varphi_{j}:=\iota_{n-j-1}^{*} \psi_{j}$ lies in $C_{j-1}^{h} \mathfrak{V}_{\bullet}\left\{\Sigma^{n-j-1} \overline{\mathbf{W}}_{n}\right\}_{0}$, and by induction it represents

$$
\mathfrak{M}_{\mathcal{B}} C \Sigma^{n-j-1} \overline{\mathbf{W}}_{n} \stackrel{\left(F_{j}\right)_{*}}{\longrightarrow} C_{j-1}^{h} \mathfrak{M}_{\mathcal{B}} \mathbf{W}_{\bullet}^{[n-1]} \stackrel{C_{j-1} \mathfrak{f}^{[n-1]}}{\longrightarrow} C_{j-1}^{h} \mathfrak{V} \bullet
$$

(in the notation of (3.8)). If $q_{n-j-2}: C \Sigma^{n-j-2} \overline{\mathbf{W}}_{n} \rightarrow \Sigma^{n-j-1} \overline{\mathbf{W}}_{n}$ is the quotient map, this implies that $q_{n-j-2}^{*} \varphi_{j}$ represents

$$
\mathfrak{M}_{\mathcal{B}} \Sigma^{n-j-1} \overline{\mathbf{W}}_{n} \stackrel{\left(a_{j}\right)_{*}}{\longrightarrow} Z_{j-1}^{h} \mathfrak{M}_{\mathcal{B}} \mathbf{W}_{\bullet}^{[n-1]} \stackrel{Z_{j-1} \mathfrak{f}^{[n-1]}}{\longrightarrow} Z_{j-1}^{h} \mathfrak{V}_{\bullet}
$$

(again using the notation of (3.8)), so $q_{n-j-2}^{*} \varphi_{j}$ is in $Z_{j-1}^{h} \mathfrak{V} \bullet\left\{C \Sigma^{n-j-2} \overline{\mathbf{W}}_{n}\right\}_{0}$, Similarly, $d_{0}^{h} \varphi_{j}$ actually lies in $Z_{j-2}^{h} \mathfrak{V}_{\bullet}\left\{C \Sigma^{n-j-2} \overline{\mathbf{W}}_{n}\right\}_{0}$, and represents

$$
\mathfrak{M}_{\mathcal{B}} \Sigma^{n-j} \overline{\mathbf{W}}_{n} \stackrel{\left(a_{j-1}\right)_{*}}{\longrightarrow} Z_{j-2}^{h} \mathfrak{M}_{\mathcal{B}} \mathbf{W}_{\bullet}^{[n-1]} \stackrel{Z_{j-2} \mathfrak{f}^{[n-1]}}{\longrightarrow} Z_{j-2}^{h} \mathfrak{V} \bullet
$$

The nullhomotopy $F_{k}$ for $v_{k-1} \circ a_{k}$ (cf. (3.8)). is represented by $\varphi_{k} \in$ $C_{k-1}^{h} \mathfrak{V}_{\bullet}\left\{\Sigma^{n-k-1} \overline{\mathbf{W}}_{n}\right\}_{0}$, and as in Step B we use the embedding of $C_{k-1}^{h} \mathfrak{V}_{\bullet}\left\{\Sigma^{n-k-1} \overline{\mathbf{W}}_{n}\right\}$ in $M_{k} \mathfrak{V}_{\bullet}\left\{\Sigma^{n-k-1} \overline{\mathbf{W}}_{n}\right\}$ and the facts that $\delta_{k}: \mathfrak{V}_{k}\left\{\Sigma^{n-k-1} \overline{\mathbf{W}}_{n}\right\} \rightarrow M_{k} \mathfrak{V}_{\bullet}\left\{\Sigma^{n-k-1} \overline{\mathbf{W}}_{n}\right\}$ is a fibration, and that $\varphi_{k} \quad$ lifts up to homotopy to $\mathfrak{V}_{j}\left\{C \Sigma^{n-k-1} \overline{\mathbf{W}}_{n}\right\} \quad$ (since $C \Sigma^{n-k-1} \overline{\mathbf{W}}_{n}$ is contractible) to obtain an element $\psi_{k}$ in $\mathfrak{V}_{j}\left\{C \Sigma^{n-k-1} \overline{\mathbf{W}}_{n}\right\}_{0}$ (with $d_{i}^{h} \psi_{k}=0$ for $2 \leq i$ ). such that $\varphi_{k}:=d_{0}^{h} \psi_{k}$.

Step D. The three conditions (3.11)- 3.12)- 3.13) on $\varphi_{j}:=d_{0}^{h} \psi_{j} \quad(0 \leq j \leq n)$ are all that is needed in order for the elements $\psi_{j}$ to fit together to define a map of restricted simplicial $\mathcal{B}$-presheaves $\widetilde{\mathfrak{f}}^{[n-1]}: \mathfrak{M}_{\mathcal{B}} \widetilde{\mathbf{W}}_{\bullet}^{[n]} \rightarrow i^{*} \mathfrak{V}_{\bullet}$ extending $i^{*} \mathfrak{f}^{[n-1]}$ (in the notation of 90.4 , and so, using (3.9), an induced map of simplicial $\mathcal{B}$-presheaves $\widehat{\mathfrak{f}}^{[n-1]}: \mathfrak{M}_{\mathcal{B}} \widehat{\mathbf{W}}_{\bullet}^{[n]} \rightarrow \mathfrak{V}_{\bullet}$, which is a levelwise weak equivalence through dimension $n$. 
Recall from [BJT2, 2.C] that $\mathbf{W}_{\bullet}^{[n]}$ is constructed by the following factorizations in the Reedy model category structure on $\mathcal{S}_{*}^{\Theta_{\mathcal{B}}^{\mathrm{op}} \times \Delta^{\mathrm{op}}} \quad$ (see $\sqrt{2.12}$ ):

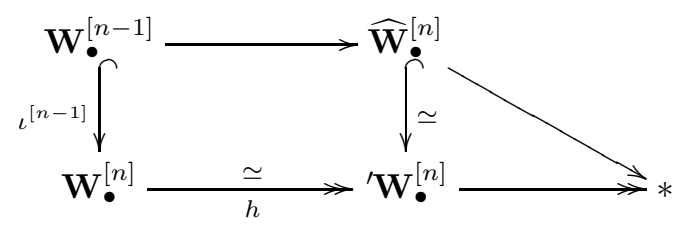

where $\hookrightarrow$ indicates a cofibration and $\longrightarrow$ a fibration, with the top horizontal map a levelwise weak equivalence in simplicial dimensions $\leq n-1$, so the same is true of the left vertical map.

Applying $\mathfrak{V}_{\bullet}\{-\}$ to (3.14) yields a diagram of bisimplicial spaces, and taking diagonals, a similar diagram in $\mathcal{S}_{*}^{\Delta^{\mathrm{op}}}$. Since by our initial assumption all objects of (3.14), in each simplicial dimension, are in $\boldsymbol{\Theta}_{\mathcal{B}}$, , by Lemma 1.8 we obtain an analogous diagram of mapping spaces of $\mathcal{B}$-presheaves into $\mathfrak{V}_{\bullet}$. The sequence of elements in the simplicial set $\operatorname{diag} \mathfrak{V} \bullet\left\{\mathbf{W}_{\bullet}^{[n-1]}\right\}_{0}$ in the upper left corner corresponding to $\mathfrak{f}^{[n-1]}: \mathfrak{M}_{\mathcal{B}} \mathbf{W}_{\bullet}^{[n-1]} \rightarrow \mathfrak{V}_{\bullet}$ map by construction to the sequence in $\operatorname{diag} \mathfrak{V}_{\bullet}\left\{\widehat{\mathbf{W}}_{\bullet}^{[n]}\right\}_{0} \quad$ corresponding to $\widehat{\mathfrak{f}}^{[n-1]}$, mapping forward to a sequence $\beta$ corresponding to ${ }^{\prime}\left[{ }^{[n]}: \mathfrak{M}_{\mathcal{B}}{ }^{\prime} \mathbf{W}_{\bullet}^{[n]} \rightarrow \mathfrak{V}_{\bullet}\right.$. Since the map $h$ in (3.14) is a trivial fibration, and these are preserved by evaluation of $\mathfrak{V}_{\bullet}$ and diagonals, we see that the induced map of simplicial sets $h_{*}: \operatorname{diag} \mathfrak{V}_{\bullet}\left\{\mathbf{W}_{\bullet}^{[n]}\right\}_{0} \rightarrow \operatorname{diag} \mathfrak{V}_{\bullet}\left\{\mathbf{W}_{\bullet}^{[n]}\right\}_{0}$ is a trivial fibration. We can therefore lift $\beta$ to a sequence representing the required map $\mathfrak{f}^{[n]}: \mathfrak{M}_{\mathcal{B}} \mathbf{W}_{\bullet}^{[n]} \rightarrow \mathfrak{V}_{\bullet}$, completing the outer induction step.

3.15. Remark. The same result holds if we replace $\mathcal{B}$-presheaves by $r$-truncated $\mathcal{B}$ presheaves, since (as noted in 1.23), Lemma 1.8 still holds, and $\mathbf{W}_{\bullet}:=P^{r} \mathfrak{M}_{\mathcal{B}} \mathbf{W}_{\bullet}$ is free in each simplicial dimension.

3.16. Summary. Assume given a homotopy functor $\mathbf{T}: \mathcal{G} \rightarrow \mathcal{M}$, inducing $\mathfrak{T}:=$ $\mathbf{T} \circ N: \operatorname{sMap}_{\mathrm{St}} \rightarrow \mathcal{M}$ as in Corollary 2.14 Let $\mathcal{D}:=\mathcal{S}_{*}^{\Theta_{\mathrm{St}}^{\mathrm{op}} \times \Delta^{\mathrm{op}}}$ and $\mathcal{E}:=\mathcal{M}^{\Delta^{\mathrm{op}}}$, with the resolution model category structure on $\mathcal{D}$ determined by $\mathcal{B}$ for $\mathcal{G}$ as in $\$ 2.12$ with respect to the structure of $\$ 1.20$ for $\mathcal{S}_{*}^{\Theta_{\mathrm{St}}^{\mathrm{op}}} \quad$ (with $E^{s}$-weak equivalences on $\mathcal{E}$ ).

In the notation of $\$ 3.1$, let $\mathcal{C}$ denote the category of simplicial strict $\mathcal{B}$-mapping algebras in $\mathcal{D}$ associated to sequential realizations as in 3.5 , let $\mathcal{W}^{\prime}$ be the Reedy weak equivalences in $\mathcal{D}$, and let $\mathcal{D}_{\mathcal{C}}$ be the full subcategory hosMap $\mathrm{st}_{\mathrm{St}}$ of objects in $\operatorname{ho}\left(\mathcal{S}_{*}^{\Theta_{\mathrm{St}}^{\mathrm{op}} \times \Delta^{\mathrm{op}}}\right)$ weakly equivalent to a constant simplicial object on $\mathrm{sMap}_{\mathrm{St}}$. The

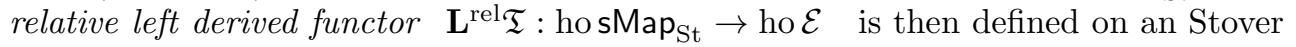
mapping algebra $\mathfrak{X}$ (more formally, on $c(\mathfrak{X}) \bullet$ ) by

(a) Choosing a simplicial resolution $\boldsymbol{\eta}: \mathfrak{V}_{\bullet} \rightarrow \mathfrak{X}$ in $\mathcal{S}_{*}^{\Theta_{B}^{\text {op }} \times \Delta^{\text {op }}}$;

(b) Choosing a CW basis $\left\{\bar{V}_{n}\right\}_{n=0}^{\infty}$ for the $\Pi_{\mathcal{B}}$-algebra-resolution $V_{\bullet}:=\pi_{0} \mathfrak{V} \bullet \rightarrow$ $\pi_{0} \mathfrak{X}$, a sequential realization $\mathcal{W}$ of $V_{\bullet}$ for $\mathbf{Y}:=N \mathfrak{X}$, with an $E^{1}$-weak equivalence $\mathfrak{M}_{\mathrm{St}} \mathbf{W}_{\bullet} \rightarrow \mathfrak{V}_{\bullet}$;

(c) Defining $\left(\mathbf{L}^{\text {rel } \mathfrak{T}}\right) \mathfrak{X}$ to be the simplicial object $\mathfrak{T M M}_{\mathrm{St}} \mathbf{W} \bullet$ in ho $\mathcal{D}^{\Delta^{\text {op }}}$ (uniquely determined up to $E^{1}$-weak equivalence). 


\section{B. Relative derived functors of dual mapping algebras}

For a given commutative ring $R$, let $\Theta^{R}=s \mathcal{M}_{\lambda}^{R}$ be the full subsimplicial category of $\mathcal{C}=\mathcal{S}_{R}$ consisting of all simplicial $R$-modules of cardinality $<\lambda$, as in 2.15 and $\mathfrak{X}=\mathfrak{M}^{R} \mathbf{Y}$ for some $\mathbf{Y} \in \mathcal{C} \quad$ (the cardinal $\lambda$ we choose may depend on $\mathbf{Y}$ ). Essentially, we may dualize the results of 93 A to this situation. Note that because $\mathbf{Y} \mapsto H^{*}(\mathbf{Y} ; R)$ is contravariant the category $\Pi^{\mathcal{A}}$-Alg resembles $\Pi_{\mathcal{B}}$-Alg in being a category of graded universal algebras, so the resolutions we need for the $\Pi^{\mathcal{A}}$-algebra $\Lambda=H^{*}(\mathbf{Y} ; R)$ will be simplicial, rather than cosimplicial, and we can use the notion of a CW resolution $V_{\bullet} \rightarrow \Lambda$ as in 3.3. However, only when $R$ is a field do we know that any free simplicial resolution in $\Pi^{\mathcal{A}}-\mathrm{Alg}^{\Delta^{\mathrm{op}}}$ has a CW basis $\left(\bar{V}_{n}\right)_{n=0}^{\infty}$ of free $\Pi^{\mathcal{A}}$-algebras (see [B13, Proposition 3.12]). For the cosimplicial resolutions of spaces, we need to dualize 3.3 as follows:

3.17. Definition. If $\mathcal{C}$ is cocomplete, the $n$-th Moore cochain object of a cosimplicial object $G^{\bullet} \in \mathcal{C}^{\Delta} \quad$ is $\quad C^{n} G^{\bullet}:=\operatorname{Coker}\left(\coprod_{i=1}^{n-1} G^{n} \stackrel{\perp_{i} d^{i}}{\longrightarrow} G^{n}\right)$, with differential $\delta^{n-1}: C^{n-1} G^{\bullet} \rightarrow C^{n} G^{\bullet} \quad$ induced by $d_{n-1}^{0}$, and structure map $v^{n}: G^{n} \rightarrow C^{n} G^{\bullet}$. We denote the cofiber of $\delta^{n-1}$ by $Z^{n} G^{\bullet}$, with structure map $w^{n}: C^{n} G^{\bullet} \longrightarrow Z^{n} G^{\bullet}$, and note that $\delta^{n-1}$ factors as $\bar{d}_{n-1}^{0} \circ w^{n-1}$.

3.18. Dual sequential realizations. Let $R$ be a commutative ring and $\lambda$ a limit cardinal, with $\Theta^{\mathcal{A}}:=\pi_{0} \Theta^{R}$ for $\Theta^{R}=s \mathcal{M}_{\lambda}^{R}$. Assume given an $R$-good space $\mathbf{Y} \in \mathcal{S}_{*}$ and a $\mathrm{CW}$ resolution $V_{\bullet}$ of the $\Pi^{\mathcal{A}}$-algebra $\Lambda=\pi_{*}^{\mathcal{A}} \mathbf{Y}$, with $\mathrm{CW}$ basis $\left\{\bar{V}_{n}\right\}_{n=0}^{\infty}$, such that for each $n \geq 0, \quad \bar{V}_{n} \cong \pi_{*}^{\mathcal{A}} \overline{\mathbf{W}}^{n}$ for some $\overline{\mathbf{W}}^{n} \in \mathbf{\Theta}^{R}$.

We define a (dual) sequential realization of $V_{\bullet}$ for $\mathbf{Y}$ to be a sequence $\mathcal{W}$ of maps

$$
\ldots \mathbf{W}_{[n+1]}^{\bullet} \stackrel{p_{[n+1]}}{\longrightarrow} \mathbf{W}_{[n]}^{\bullet} \stackrel{p_{[n]}}{\longrightarrow} \mathbf{W}_{[n-1]}^{\bullet} \ldots \mathbf{W}_{[1]}^{\bullet} \stackrel{p_{[1]}}{\longrightarrow} \mathbf{W}_{[0]}^{\bullet}
$$

between Reedy fibrant and cofibrant objects in $\mathcal{S}_{*}^{\Delta}$, such that for each $n \geq 0$ :

(i) There is an $n$-skeletal restricted cosimplicial object $\widetilde{\mathbf{W}}_{[n]}^{\cdot}$ with $\widetilde{\mathbf{W}}_{[n]}^{k}=$ $\mathbf{W}_{[n-1]}^{k} \times P \Omega^{n-k-1} \overline{\mathbf{W}}^{n} \quad$ for $0 \leq k \leq n$, where as before by convention $\Omega^{0} \overline{\mathbf{W}}^{n}=P \Omega^{-1} \overline{\mathbf{W}}^{n}=\overline{\mathbf{W}}^{n}$.

(ii) The coface map $d^{0}: C^{k} \rightarrow \widetilde{\mathbf{W}}_{[n]}^{k+1} \quad$ into the factor $P \Omega^{n-k-2} \overline{\mathbf{W}}^{n} \quad$ is the map $F^{k}$ in the commuting diagram

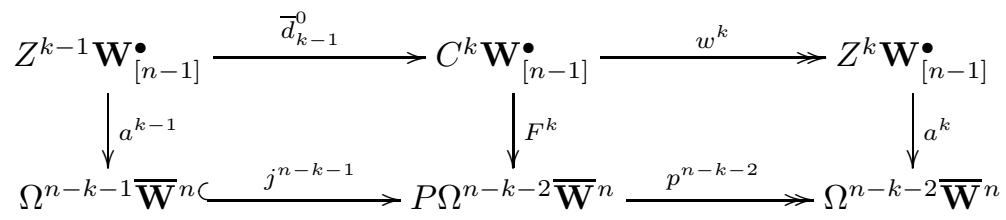

(in the notation of 93.17 ). The first coface map $d^{1}$ into $P \Omega^{n-k-2} \overline{\mathbf{W}}^{n}$ is the composite of the projection onto $P \Omega^{n-k-1} \overline{\mathbf{W}}^{n}$ with $j^{n-k-1} \circ p^{n-k-1}$, and $d^{i}$ into the factor $P \Omega^{n-k-2} \overline{\mathbf{W}}^{n}$ is zero for $i>1$.

We start with a realization of the $n$-th attaching map $\partial_{n}^{V}: \bar{V}_{n} \rightarrow C_{n-1} V$ • for the given CW resolution as our choice for $F^{n-1}: C^{n-1} \mathbf{W}_{[n-1]}^{\bullet} \rightarrow \overline{\mathbf{W}}^{n}$. 
(iii) Let $\widehat{\mathbf{W}}_{\bullet}^{[n]}$ be the pullback of $\mathbf{W}_{[n-1]}^{\bullet} \leftarrow \mathcal{F} i^{*} \mathbf{W}_{[n-1]}^{\bullet} \rightarrow \mathcal{F} \widetilde{\mathbf{W}}_{\bullet}^{[n]}$, where $\mathcal{F}: \mathcal{C}^{\Delta} \rightarrow \mathcal{C}^{\Delta}$ is the right adjoint of the forgetful functor $i^{*}: \mathcal{C}^{\Delta} \rightarrow \mathcal{C}^{\Delta_{+}}$ (see 40.4 ), with $\mathbf{W}_{[n]}^{\bullet} \quad$ a Reedy fibrant and cofibrant replacement for $\widehat{\mathbf{W}}_{\bullet}^{[n]}$.

Again, $\mathbf{W}^{\bullet}:=\lim _{n} \mathbf{W}_{[n]}^{\bullet}$ is a cosimplicial resolution of $\mathbf{Y}$ in the resolution model category $\mathcal{C}^{\Delta}$, and in fact, the sequential realization $\mathcal{W}$ can be constructed starting from any $R$-mapping algebra $\mathfrak{X}$. See [BS1, $\S 2 \&$ Appendix A] for further details.

The proof of Theorem 3.10 can be dualized to yield:

3.21. Theorem. Given a commutative ring $R$ with $\Theta^{\mathcal{A}}=s \mathcal{M}_{\lambda}^{R} \quad$ and an $R$-good space $\mathbf{Y}$, let $\boldsymbol{\eta}: \mathfrak{V}_{\bullet} \rightarrow \mathfrak{X}=\mathfrak{M}^{R} \mathbf{Y} \quad$ be a simplicial resolution in $\mathcal{S}_{*}^{\Theta^{R} \times \Delta^{\text {op }}}$ with a $C W$ basis $\left\{\bar{V}_{n}\right\}_{n=0}^{\infty}$ for the $\Pi^{\mathcal{A}}$-algebra-resolution $V_{\bullet}:=\pi_{0} \mathfrak{V}_{\bullet} \rightarrow \Lambda=\pi_{*}^{\mathcal{A}} \mathbf{Y}$. Then for any sequential realization $\mathcal{W}$ of $V_{\bullet}$ for $\mathbf{Y}$, there is a Reedy weak equivalence of simplicial dual weak $\mathcal{A}$-mapping algebras $\mathfrak{f}: \mathfrak{W}_{\bullet}:=\mathfrak{M}^{\mathcal{A}} \mathbf{W}^{\bullet} \rightarrow \mathfrak{V}_{\bullet}$.

The dual of Remark 3.15, for the $n$-truncated case, also holds.

3.22. Summary. Given a functor $\mathfrak{T}: \mathrm{sMap}_{\mathrm{re}}^{\mathrm{St}, R} \rightarrow \mathcal{D}$ as in Corollary 2.23, the relative right derived functor $\mathbf{R}^{\text {rel }} \mathfrak{T}$ : hosMap $\mathrm{se}_{\mathrm{re}, R}^{\mathrm{St}} \rightarrow \mathrm{ho}\left(\mathcal{D}^{\Delta}\right)$ applied to $\mathfrak{X}:=\mathfrak{M}^{\mathrm{St}, R} \mathbf{Y}$ for $R$-good $\mathbf{Y} \in \mathcal{S}_{*}$, is obtained by

(a) Choosing a simplicial resolution $\boldsymbol{\eta}: \mathfrak{V}_{\bullet} \rightarrow \mathfrak{X}$ in the model category $\mathcal{S}_{*}^{\Theta^{\mathcal{A}} \times \Delta^{\mathrm{op}}}$

(b) Assuming the $\Pi^{\mathcal{A}}$-algebra-resolution $V_{\bullet}:=\pi_{0} \mathfrak{V}_{\bullet} \rightarrow \pi_{*}^{\mathcal{A}} \mathbf{Y}$ has a CW basis $\left\{\bar{V}_{n}\right\}_{n=0}^{\infty} \quad$ (e.g., if $R$ is a field), choosing a sequential realization $\mathcal{W}$ of $V_{\bullet}$;

(c) Defining $\left(\mathbf{R}^{\text {rel }} \mathfrak{T}\right) \mathfrak{X}$ to be the cosimplicial object $\mathfrak{T M}^{\mathrm{St}, R} \mathbf{W}^{\bullet}$ in $\mathcal{D}^{\Delta}$.

3.23. Example. For $\mathbf{Z} \in \mathcal{S}_{*}$ and $\mathbf{T}:=\operatorname{map}_{*}(\mathbf{Z},-)$ as in 22.24 , if $\mathfrak{Z}=\mathfrak{M}^{\mathrm{St}, R} \mathbf{Z}$ and $\mathfrak{X}=\mathfrak{M}^{\mathrm{St}, R} \mathbf{Y} \quad$ for some $R$-good space $\mathbf{Y}$, and $\mathfrak{V}_{\bullet}=\mathfrak{M}^{\mathrm{St}, R} \mathbf{W}^{\bullet} \quad$ for some cosimplicial resolution $\mathbf{Y} \rightarrow \mathbf{W}^{\bullet}$, then $\left(\mathbf{R}^{\text {rel } \mathfrak{T}}\right) \mathfrak{X}:=\mathfrak{T} \mathfrak{V} \bullet$ is the cosimplicial space $\mathfrak{Z}\left\{\mathbf{W}^{\bullet}\right\} \quad$ (up to $E^{2}$-equivalence).

\section{TRUnCATING HigheR ORDER DERIVED FUnCtORS}

So far we have shown only that the usual total derived functor LT of a continuous functor $\mathbf{T}: \mathcal{C} \rightarrow \mathcal{D}$ can be interpreted (under suitable assumptions) as derived functors of the corresponding mapping algebras. Although there are many technicalities involved, the result is hardly surprising, since, under these assumptions, mapping algebras carry the same homotopy information as objects in $\mathcal{C}$ (Theorems 2.13 and 2.22).

The point is that mapping algebras are the right framework for truncating the homotopy information (using Postnikov sections), while still retaining enough to compute the required term in the homotopy spectral sequences for $\mathbf{T W} \bullet$ or $\mathbf{T W}^{\bullet}$.

\section{A. Truncating derived functors of mapping algebras}

Not every homotopy functor $\mathbf{T}$ (and the corresponding $\mathfrak{T}$ ) will behave as we want with respect to such truncation. We therefore require the following:

4.1. Definition. For any $2 \leq r \leq \infty$, let $\mathcal{E}^{r}$ denote the category of $r$-truncated homological spectral sequences $\left\{E_{* *}^{k}\right\}_{k=1}^{r}$, equipped with a differential $d^{r}: E_{t, i}^{r} \rightarrow$ 
$E_{t-r-1, i+r}^{r}$, which need not satisfy $d^{r} \circ d^{r}=0$. A map in $\mathcal{E}^{r}$ is called a weak equivalence if it induces an isomorphism in $E_{* *}^{2}$ (and thus also for $r \leq k>2$ ). This defines the corresponding localized category ho $\mathcal{E}^{r}$. We have truncation functors $P^{r}: \mathcal{E}^{n} \rightarrow \mathcal{E}^{r}$ for each $r \leq n \leq \infty$. Note that the homotopy spectral sequence of a simplicial space defines a homotopy functor $\mathcal{S}^{\infty}: \mathcal{G}^{\Delta^{\mathrm{op}}} \rightarrow \mathcal{E}^{\infty}$ (with respect to $E^{2}$-equivalences in the source and target), and write $\mathcal{S}^{r}:=P^{r} \circ \mathcal{S}^{\infty}$.

4.2. Definition. Any homotopy functor $\mathbf{T}: \mathcal{G} \rightarrow \mathcal{G}$, and the corresponding $\mathfrak{T}$ :

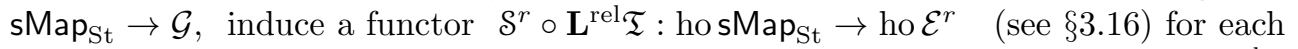
$r \geq 2$. We say that $\mathbf{T}$ (and $\mathfrak{T}$ ) are level if for every $r \geq 2$, this functor $\mathcal{S}^{r} \circ \mathbf{L}^{\text {rel } \mathfrak{T}}$ factors through a functor $\mathbf{L}^{\text {rel } \mathfrak{T}^{r-2}}:$ hosMap $\mathrm{St}_{\mathrm{St}}^{r-2} \rightarrow$ ho $\mathcal{E}^{r}$.

Here hosMap St $^{n}$ is the subcategory of $\operatorname{ho}\left(\mathcal{S}_{[n]}^{\Theta_{\mathrm{St}}^{\mathrm{op}} \times \Delta^{\mathrm{op}}}\right)$ weakly equivalent to $c(\mathfrak{X})_{\bullet}$, for $\mathfrak{X}$ in the subcategory $\operatorname{sMap}_{\mathrm{St}}^{n}$ of $n$-truncated Stover mapping algebras (cf. \$1.23).

In order to identify which homotopy functors are level, we shall need the following notion introduced in [BB1, §1] (see also [BDG]):

4.3. Definition. Let $\mathcal{C}$ be $\operatorname{Top}_{0}, \quad \mathcal{S}_{*}$, or $\mathcal{G}$ : for any $n \geq 0$, an $n$-stem in $\mathcal{C}$ is a tower:

$$
\mathcal{Q}:=\left(\ldots \rightarrow Q_{k+1} \stackrel{q_{k+1}}{\longrightarrow} Q_{k} \stackrel{q_{k}}{\longrightarrow} Q_{k-1} \ldots Q_{1}\right)
$$

in $\mathcal{C}^{(\mathbb{N}, \leq)}$, in which $\pi_{i}\left(Q_{k}\right)=0$ for $i<k$ or $i>n+k$, and $\pi_{i} q_{k}$ is an isomorphism for $k \leq i<n+k$. Here $(\mathbb{N}, \leq)$ is the usual linearly ordered category of the natural numbers. The object $Q_{k} \in \mathcal{C}$ is called the $k$-th $n$-window of $\mathcal{Q}$.

We denote by Stem $[n]$ the full subcategory of $n$-stems in the functor category $\mathcal{C}^{(\mathbb{N}, \leq)}$, with the model category structure on the latter as in $[\underline{\mathrm{H}}, 11.6]$. The Postnikov $n$-stem functor $\mathcal{P}[n]: \mathcal{C} \rightarrow \operatorname{Stem}[n]$ is given by $\mathcal{P}[n] X:=\left\{P^{n+k+1} X\langle k\rangle\right\}_{k=1}^{\infty}$.

To avoid the need to distinguish the cases $\mathcal{C}=$ Top $_{0}$ or $\mathcal{G}$, we everywhere use the Top-indexing for spheres, homotopy groups, Postnikov systems, and connected covers (as in 2.1 ).

By [BB1, Theorem 4.13 \& Corollary 4.16] we have:

4.5. Theorem. For each $r \geq 2$ there is a functor $\hat{\mathcal{S}}^{r}: \operatorname{Stem}[r-1]^{\Delta^{\mathrm{op}}} \rightarrow \mathcal{E}^{r}$ which associates to any simplicial $(r-1)$-stem $Q_{\bullet} \quad$ an $r$-truncated spectral sequence. Moreover, $\quad \hat{\mathcal{S}}^{r} \circ \mathcal{P}[r-1]: \mathcal{C}^{\Delta^{\mathrm{op}}} \rightarrow \mathcal{E}^{r}$ is naturally equivalent to $\mathcal{S}^{r}$, so when $Q_{\bullet}=\mathcal{P}[r-1] \mathbf{X} \bullet \quad$ this is the truncation of the usual homotopy spectral sequence for X.. In this case we have $d^{r} \circ d^{r}=0$, so in fact the spectral sequence is determined through $E_{* *}^{r+1}$ (though without $d_{r+1}$ ).

4.6. Corollary. A functor $\mathfrak{T}: \operatorname{sMap}_{\mathrm{St}} \rightarrow \mathcal{G}$ associated to a homotopy functor $\mathbf{T}: \mathcal{G} \rightarrow \mathcal{G}$ is level if for each $r \geq 1$, the relative derived functor $\mathcal{S}^{r} \circ \mathbf{L}^{\text {rel } \mathfrak{T}}$ : ho sMap $\rightarrow \mathcal{E}^{r}$ factors as $\hat{\mathcal{S}}^{r} \circ \mathbf{L}^{\text {rel } \mathfrak{T}^{r-1}}$ for some functor $\mathbf{L}^{\text {rel } \mathfrak{T}^{r-1}}$ : ho sMap $\mathrm{St}^{r-1} \rightarrow$ ho(Stem $\left.[r-1]^{\Delta^{\mathrm{op}}}\right)$.

In order for Corollary 4.6 to be of any use, we must identify level homotopy functors $\mathbf{T}$ for which the homotopy spectral sequence of $\mathbf{T X}$. is of interest. We first note: 
4.7. Lemma. For $\mathcal{B}$ as in 1 1.4, any n-truncated weak $\mathcal{B}$-mapping algebra $\mathfrak{X} \in \mathrm{sMap}_{\mathrm{St}}^{n}$ is functorially realizable by an n-stem $\mathcal{Q}=\left\{Q_{k}\right\}_{k=1}^{\infty}$. Moreover, if $\mathfrak{X}=P^{n} \mathfrak{M}_{\mathcal{B}} \mathbf{Y}$ for some $\mathbf{Y} \in \mathcal{G}$, then $\mathcal{Q}$ is naturally weakly equivalent to the Postnikov n-stem $\mathcal{P}[n] \mathbf{Y}$.

Proof. This result appears in [BB2, §10.5] for Stover mapping algebras, but in fact we need only observe that for $n \geq 1$, the action of $P^{n} \boldsymbol{\Theta}_{\mathcal{B}}$ on $\mathfrak{X}$ includes inter alia an $A_{n}$-structure on $X_{k}:=\mathfrak{X}\left\{\mathbf{S}^{k}\right\}$, so allowing $P^{n} X_{k}$ to be delooped to produce the window $Q_{k}$ by [Sta, Corollary 11.12]. The weak equivalences (1.25), together with [Ma1, Theorem 12.7], yield the structure maps for the $n$-stem $\mathcal{Q}$

The simplest example is from [Bl1], where it is used to construct a spectral sequence for computing $H_{*} \mathbf{Y}$ from the $\Pi$-algebra $\pi_{*} \mathbf{Y}$ :

4.8. Proposition. The abelianization functor $\mathrm{Ab}: \mathcal{G} \rightarrow \mathcal{G}$ is level.

Proof. Let $\mathcal{Q}=\left\{Q_{k}\right\}_{k=1}^{\infty} \quad$ denote the Postnikov $n$-stem of a space $\mathbf{X}$, and $\mathcal{R}=$ $\left\{R_{k}\right\}_{k=1}^{\infty}$ that of $\mathrm{Ab} \mathbf{X}$. Note that for each $k \geq 0$, the covering map $\rho: \mathbf{X}\langle k\rangle \rightarrow \mathbf{X}$ induces a map $\rho_{*}: \mathrm{Ab}(\mathbf{X}\langle k\rangle) \rightarrow \mathrm{Ab} \mathbf{X}$, which factors through $(\mathrm{Ab} \mathbf{X})\langle k\rangle$ by cellularity (uniquely, if we choose a $(k+1)$-reduced model for connected covers which is an inclusion of a sub-simplicial group, in $\mathcal{G}$ ). Furthermore, by the Hurewicz Theorem, for each $m \geq 0$ the structure map $p_{m}: \mathbf{X} \rightarrow P^{m} \mathbf{X}$ induces an isomorphism $H_{i} \mathbf{X} \rightarrow H_{i} P^{m} \mathbf{X} \quad$ for $i \leq m$, and an epimorphism $H_{m+1} \mathbf{X} \rightarrow$ $H_{m+1} P^{m} \mathbf{X}$, so the natural map $P^{m}(\mathrm{Ab} \mathbf{X}) \rightarrow P^{m} \mathrm{Ab}\left(P^{m} \mathbf{X}\right)$ is a weak equivalence. Thus we have a natural weak equivalence $P^{n+k+1}\left(\mathrm{Ab} Q_{k}\right) \simeq R_{k}$ for each $k \geq 0$.

Thus a given a simplicial resolution $\mathfrak{V}_{\bullet} \rightarrow P^{n} \mathfrak{X}=P^{n} \mathfrak{M}_{\mathcal{B}} \mathbf{Y}$ of $n$-truncated $\mathcal{B}$-presheaves in the model category $\mathcal{S}_{[n]}^{\Theta_{\mathcal{B}}^{\text {op }}}$, by Lemma 4.7, we obtain a simplicial $n$-stem $\mathcal{Q}_{\bullet}$, which yields in turn the required simplicial $n$-stem $\mathcal{R}_{\bullet}:=\mathcal{P}[n]\left(\mathrm{Ab} \mathcal{Q}_{\bullet}\right)$.

Here are two additional examples from [Sto. The first is used to construct a spectral sequence for computing $\pi_{*} \Sigma \mathbf{Y}$ from $\pi_{*} \mathbf{Y}$ :

4.9. Proposition. The suspension functor $\Sigma: \mathcal{G} \rightarrow \mathcal{G}$ is level.

Proof. For each $n \geq 1$, any $n$-truncated weak $\mathcal{B}$-mapping algebra has a corresponding $n$-stem $\mathcal{Q}$ by Lemma 4.7 and the $\Pi$-algebra $\Lambda:=\pi_{0} \mathfrak{X}$ determines the $\Pi$-algebra structure on $\pi_{*} Q_{k}$ for each $k \geq 0$. If $\mathfrak{X} \simeq P^{n} \mathfrak{M}_{\mathcal{B}} \mathbf{X}$ for some space $\mathbf{X}$, then $\Lambda$ is isomorphic to $\pi_{*} \mathbf{X}$ and $Q_{k} \simeq P^{n+k+1} \mathbf{X}\langle k\rangle$. To understand $\mathbf{L T}$, we need only consider the case when $\Lambda$ is a free $\Pi$-algebra.

Now let $\mathcal{R}=\left\{R_{k}\right\}_{k=1}^{\infty}$, denote the Postnikov $n$-stem of $\Sigma \mathbf{X}$. As in the proof of Proposition 4.8, the covering map $\rho: \mathbf{X}\langle k\rangle \rightarrow \mathbf{X}$ induces a map $\rho_{*}: \Sigma(\mathbf{X}\langle k\rangle) \rightarrow$ $(\Sigma \mathbf{X})\langle k+1\rangle$. Taking Postnikov sections yields natural maps $p_{k}: P^{n+k+2}\left(\Sigma Q_{k}\right) \rightarrow$ $R_{k+1}$. In particular, $p_{0}: P^{n+2}\left(\Sigma Q_{0}\right) \rightarrow R_{1}=P^{n+1}(\Sigma \mathbf{X})$ is a weak equivalence by the Hurewicz Theorem, with $P^{1} R_{1} \simeq \mathbf{X}_{1} \quad$ (a wedge of 1-spheres, and thus aspherical).

However, for $k>1$ there is no functorial description of $R_{k}$ in terms of $\mathcal{Q}$. Thus if $\mathfrak{T}:=\Sigma \circ N: \operatorname{sMap}_{\mathrm{St}} \rightarrow \mathcal{G}$ is induced by $\Sigma: \mathcal{G} \rightarrow \mathcal{G}$ as in Corollary 2.14 in order to define $\mathbf{L T}^{n}:$ ho sMap ${ }_{\mathrm{St}}^{n} \rightarrow \operatorname{Stem}[n]$ we must proceed as follows: 
By Lemma 4.7 a simplicial resolution $\mathfrak{V}_{\bullet} \rightarrow P^{n} \mathfrak{X}=P^{n} \mathfrak{M}_{\mathcal{B}} \mathbf{Y}$ of $n$-truncated weak $\mathcal{B}$-mapping algebras yields a simplicial $n$-stem $\mathcal{Q}_{\bullet}$. Since the simplicial $\Pi$ algebra $V_{\bullet}:=\pi_{o} \mathfrak{V}_{\bullet} \quad$ is a free resolution of $\Lambda:=\pi_{0} \mathfrak{X}$, it has a (non-canonical) CW basis $\left\{\bar{V}_{n}\right\}_{n=0}^{\infty}$ for it, which in turn has a sequential realization $\mathcal{W}$ (see $\$ 3.5$ ). By Remark 3.15, there is a Reedy weak equivalence of simplicial $n$-truncated weak $\mathcal{B}$-mapping algebras $\mathfrak{f}: P^{n} \mathfrak{W}_{\bullet} \rightarrow \mathfrak{V}_{\bullet}$, where $\mathfrak{W}_{\bullet}$ is realizable as $\mathfrak{M}_{\mathcal{B}} \mathbf{W}_{\bullet}$. We can realize $P^{n} \mathfrak{W}_{\bullet}$ by the simplicial $n$-stem $\widehat{\mathcal{Q}}_{\bullet} \simeq \mathcal{P}[n] \mathbf{W}_{\bullet}$, and let $\Sigma \widehat{\mathcal{Q}} \bullet$ denote the simplicial $n$-stem obtained by applying $\Sigma$ to each window of $\widehat{\mathcal{Q}}_{\bullet} \quad$ (and taking appropriate Postnikov sections). If $\widehat{\mathcal{R}} \bullet \quad$ denotes the simplicial Postnikov $n$-stem $\mathcal{P}[n] \Sigma \mathbf{W}_{\bullet}$, we have a map of simplicial $n$-stems $\widehat{p}: \Sigma \widehat{\mathcal{Q}}_{\bullet} \rightarrow \widehat{\mathcal{R}}_{\bullet}$, as explained above.

Similarly, the simplicial $n$-truncated $\mathcal{B}$-presheaf $\mathfrak{V} \bullet \quad$ yields a simplicial $n$-stem $\mathcal{Q}_{\bullet}$, and $\mathfrak{f}: P^{n} \mathfrak{W}_{\bullet} \rightarrow \mathfrak{V}_{\bullet} \quad$ induces a levelwise weak equivalence of simplicial $n$ stems $\widehat{f}: \Sigma \widehat{\mathcal{Q}} \bullet \rightarrow \Sigma \mathcal{Q} \bullet \quad$ (in the Reedy model structure). We may assume that each window of all the simplicial $n$-stems described here are cofibrant in $\mathcal{G}$, so they are Reedy cofibrant. Thus if we let $\mathcal{R} \bullet \quad$ denote the homotopy pushout of $\widehat{f}$ and $\widehat{p}$ (in the Reedy model category of simplicial $\mathcal{B}$-presheaves), we have a Reedy weak equivalence $\widehat{\mathcal{R}}_{\bullet} \rightarrow \mathcal{R}_{\bullet} \quad($ cf. $[\mathrm{H}$, Proposition 13.1.2]), as well as a structure map of simplicial $n$-stems $p: \mathcal{Q}_{\bullet} \rightarrow \mathcal{R}_{\bullet}$.

We define $\left(\mathbf{L} \mathfrak{T}^{n}\right) P^{n} \mathfrak{X}$ to be the simplicial $n$-stem $\mathcal{R}$. To see that $\mathbf{L T}^{n}$ is welldefined, replace $\mathfrak{V}_{\bullet}$ by some other simplicial resolution $\mathfrak{U}_{\bullet} \rightarrow P^{n} \mathfrak{X}$ of $n$-truncated $\mathcal{B}$-presheaves, with $\mathcal{Z}$ a sequential realization of $\pi_{0} \mathfrak{U}_{\bullet} \quad$ for $\mathbf{Y}$. Let $\mathcal{R} \bullet$ and $\mathcal{S}_{\bullet}$ denote the simplicial $n$-stems associated as above to $\mathfrak{V}_{\bullet}$ and $\mathfrak{U}_{\bullet}$ respectively. We then have a weak equivalence of simplicial spaces $g: \mathbf{W}_{\bullet} \rightarrow \mathbf{Z}_{\bullet} \quad$ in the resolution model category structure with respect to $\boldsymbol{\Theta}_{\mathcal{B}}$ (since both are cofibrant replacements for $\left.c(\mathbf{Y})_{\bullet}\right)$, and this will induce a weak equivalence $\mathfrak{V}_{\bullet} \rightarrow \mathfrak{U}_{\bullet}$ in the resolution model structure of $\$ 2.12$, and thus the same holds for the simplicial $n$-stems $\mathcal{R}_{\bullet}$ and $\mathcal{S}$ • (cf. [Sto, Theorem 1.9]).

The next example is used to construct a van Kampen spectral sequence to compute $\pi_{*}(\mathbf{Y} \vee \mathbf{Z})$ from $\pi_{*} \mathbf{Y}$ and $\pi_{*} \mathbf{Z}$ :

4.10. Proposition. The wedge bifunctor $\vee: \mathcal{G} \times \mathcal{G} \rightarrow \mathcal{G}$ is level.

Proof. The proof is entirely analogous to that of Proposition 4.9, given two Stover mapping algebras $\mathfrak{X}$ and $\mathfrak{Y}$, realizable by $\mathbf{Y}$ and $\mathbf{Z}$, respectively, their $n$-truncations are realizable by $n$-stems $\mathcal{Q}$ and $\mathcal{S}$, weakly equivalent to the Postnikov $n$-stem $\mathcal{P}[n] \mathbf{Y}$ and $\mathcal{P}[n] \mathbf{Z}$, respectively. Once again we cannot reconstruct the Postnikov $n$-stem for $\mathbf{Y} \vee \mathbf{Z}$ directly from the window-wise wedge of $\mathcal{Q}$ and $\mathcal{S}$ (except for the bottom window), but must have recourse to sequential realizations of the full simplicial resolutions.

4.11. Remark. Stover set up spectral sequences for arbitrary homotopy colimits in $\mathrm{Top}_{0}$ (see [Sto, Theorem 1.2]), and one can obtain similar results for the left derived functors appearing as the $E^{2}$-terms of these spectral sequences.

\section{4,B. Truncating derived functors of dual mapping algebras}

We may dualize Definitions 4.1 and 4.2 as follows: 
4.12. Definition. For any $2 \leq r \leq \infty$ we let $\mathcal{E}_{r}$ denote the category of $r$-truncated cohomological spectral sequences $\left\{E_{k}^{* *}\right\}_{k=1}^{r} \quad$ (again, the last differential need not satisfy $\left.d_{r} \circ d_{r}=0\right)$. A weak equivalence in $\mathcal{E}_{r}$ is a map inducing an isomorphism in $E_{2}^{* *}$. Again we have truncation functors $P^{r}: \mathcal{E}_{n} \rightarrow \mathcal{E}_{r}$. The homotopy spectral sequence of a cosimplicial space defines a homotopy functor $\mathcal{S}_{\infty}: \mathcal{S}_{*}^{\Delta} \rightarrow \mathcal{E}_{\infty}$, and we write $\mathcal{S}_{r}:=P^{r} \circ \mathcal{S}_{\infty}$.

If $\mathbf{T}: \mathcal{S}_{R} \rightarrow \mathcal{D}$ is a homotopy functor preserving $R$-equivalences, we say that $\mathbf{T}$, and the corresponding $\mathfrak{T}: \mathrm{sMap}_{\mathrm{re}}^{\mathrm{St}, R} \rightarrow \mathcal{D}$ of Corollary 2.23, are level if for any $r \geq 2$ and weakly $R$-good dual strict Stover mapping algebra $\mathfrak{X}=\mathfrak{M}^{\mathrm{St}, R} \mathbf{Y}$ (see 2.201), $\mathcal{S}_{r} \mathbf{R}^{\text {rel } \mathfrak{T X}}$ factors up to isomorphism through the $(r-2)$-truncated simplicial dual strict Stover mapping algebra $P^{r-2} \mathfrak{M}^{\mathrm{St}, R}\left(\mathbf{R}^{\text {rel }} \mathfrak{T} \mathfrak{X}\right)$, up to weak equivalence in $\mathcal{S}_{[n]}^{\Theta^{\mathcal{A}} \times \Delta^{\mathrm{op}}} \quad\left(\right.$ see $\left.\oint_{2.21}\right)$.

Although the analogue of Theorem 4.5 was also shown in BB1 to hold for the homotopy spectral sequence of a cosimplicial space, this does not appear to be helpful in showing that functors of $R$-mapping algebras are level - mainly because there is no simple connection between maps into Eilenberg-Mac Lane spaces and maps out of spheres. Thus a more direct approach is needed here.

Our main result in this connection, which may be of independent interest, is the following reinterpretation of the results of $[\mathrm{BBS}$ :

4.13. Theorem. For any $\mathbf{Z} \in \mathcal{S}_{*}$ and $R=\mathbb{F}_{p}$ or $\mathbb{Q}$, the unstable $R$-Adams spectral sequence for $\mathbf{T}:=\operatorname{map}_{*}(\mathbf{Z},-)$ applied to $\mathbf{Y} \in \mathcal{S}_{R} \quad$ (see [BK1, §7.2]) is determined by the simplicial $R$-mapping algebra $\left(\mathfrak{M}^{R} \mathbf{R}^{\text {rel }} \mathfrak{T}\right) \mathfrak{M}^{\mathrm{St}, R} \mathbf{Y}$, and $\mathbf{T}$ is level.

Proof. Let $\mathbf{Y} \rightarrow \mathbf{W}^{\bullet}$ be a cosimplicial resolution, which we may assume without loss of generality to be associated to a dual sequential realization $\mathcal{W}$ as in $₫ 3$, by Definition 3.22

We know that the homotopy spectral sequence for the cosimplicial space $\mathbf{X}^{\bullet}:=$ $\operatorname{map}\left(\mathbf{Z}, \mathbf{W}^{\bullet}\right) \quad$ is determined in principle by the simplicial dual strict $\mathcal{A}$-mapping algebra $\mathfrak{W}_{\bullet}:=\mathfrak{M}^{\mathrm{St}, R} \mathbf{W}^{\bullet}$. Following the description in BBS] (and compare [Bou1]) we now explain how this can be made explicit:

By [BBS, Proposition 4.18] the unstable Adams spectral sequence for $\mathbf{Y}$ as above agrees from the $E_{2}$-term on with that associated to the fibration sequences

$$
\Omega^{n} \overline{\mathbf{W}}^{n} \rightarrow \operatorname{Tot}_{n} \widehat{\mathbf{W}}_{[n]}^{\bullet} \rightarrow \operatorname{Tot}_{n-1} \mathbf{W}_{[n-1]}^{\bullet},
$$

in the notation of 3.18 , so the same is true of the homotopy spectral sequence for $\mathbf{X}^{\bullet}:=\operatorname{map}\left(\mathbf{Z}, \mathbf{W}^{\bullet}\right)$, if we apply $\operatorname{map}_{*}(\mathbf{Z},-)$ before taking Tot.

An element $\gamma \in E_{1}^{n, k+n}$ is thus represented by a map $\Sigma^{k} \mathbf{Z} \rightarrow \operatorname{Tot}_{n} \boldsymbol{\Sigma} D_{[n]}^{\bullet}$, where $\boldsymbol{\Sigma} D_{[n]}^{\bullet}$ is the fiber of the Reedy fibration $\widehat{\mathbf{W}}_{[n]}^{\bullet} \rightarrow \mathbf{W}_{[n-1]}^{\bullet}$ and $\operatorname{Tot}_{n} \boldsymbol{\Sigma} D_{[n]}^{\bullet} \simeq \Omega^{n} \overline{\mathbf{W}}^{n}$ (see [BBS, Proposition 4.12]). This is represented in turn by a map of cosimplicial spaces $G^{\bullet}: \boldsymbol{\Delta}^{\bullet} \ltimes \Sigma^{k} \mathbf{Z} \rightarrow \mathbf{W}_{[n]}^{\bullet}$ : (see $\widehat{3.18(i i i)) ~-~ t h a t ~ i s, ~ a ~ s e q u e n c e ~ o f ~ m a p s ~}$ $G_{[n]}^{j}: \boldsymbol{\Delta}^{j} \ltimes \Sigma^{k} \mathbf{Z} \rightarrow \mathbf{W}_{[n]}^{j} \quad$ (where we may assume $G_{[n]}^{j}=0$ for $j<n$ by [BBS, $(3.6)])$.

By [BBS, Theorem 5.9], for each $r \geq 2$ and $N:=n+r-1$, the differential $d_{r}: E_{r}^{n, k+n} \rightarrow E_{r}^{N+1, k+N}$ is defined on $\langle\gamma\rangle$ by the value $\phi: \Sigma^{k} \mathbf{Z} \rightarrow \Omega^{N} \overline{\mathbf{W}}^{N+1}$ of a certain $r$-th order $R$-cohomology operation. This operation is defined when the 
associated sequence of lower order operations vanish, so that there exists a chosen lift of $G^{\bullet}$ to $G_{[N]}: \boldsymbol{\Delta}^{\bullet} \ltimes \Sigma^{k} \mathbf{Z} \rightarrow \mathbf{W}_{[N]}^{\bullet}$.

The map $\phi$ is obtained by patching together the composite of the maps $G_{[N]}^{i}$ with the given maps $F_{[N+1]}^{j}: \mathbf{W}_{[N]}^{j} \rightarrow P \Omega^{N-j-1} \overline{\mathbf{W}}^{N+1}$ of (3.20). yielding a map from the boundary of a certain $(N+1)$-dimensional polyhedron $\mathcal{P}_{r}^{N+1}$, described in [BBS, §4.3] to $\operatorname{map}_{*}\left(\Sigma^{k} \mathbf{Z}, \overline{\mathbf{W}}^{N+1}\right)$. This is adjoint to a map $\widetilde{\phi}: \Sigma^{k} \mathbf{Z} \rightarrow \Omega^{N} \overline{\mathbf{W}}^{N+1}$, and by [BBS, Theorem 5.10], the class

$$
[\widetilde{\phi}] \in\left[\Sigma^{k} \mathbf{Z}, \Omega^{N} \overline{\mathbf{W}}^{N+1}\right] \cong\left[\Sigma^{k-1} \mathbf{Z}, \Omega^{N+1} \overline{\mathbf{W}}^{N+1}\right] \cong E_{1}^{N+1, k+N}
$$

(using the usual $\Sigma$ - $\Omega$ adjunction on the left) represents $d_{r}\langle\gamma\rangle \in E_{r}^{N+1, k+N}$. In particular, by [BBS, Lemma 5.7], $[\widetilde{\phi}]$ vanishes if and only if $G_{[N]}$ lifts to a map $G_{[N+1]}: \boldsymbol{\Delta}^{\bullet} \ltimes \Sigma^{k} \mathbf{Z} \rightarrow \mathbf{W}_{[N+1]}^{\bullet}$.

Because we assumed that each $\overline{\mathbf{W}}^{N}$ is in $\Theta^{R}$ (see $\sqrt{3.18}$ ), the information used in defining this higher operation is encoded by $\mathfrak{W}_{\bullet}:=\mathfrak{M}^{R} \mathbf{W}^{\bullet}$ and $\mathfrak{Z}:=\mathfrak{M}^{R} \mathbf{Z}$ Furthermore, since $G_{[N]}^{j}=0$ for $j<n$, and $\mathbf{W}_{[N]}^{\bullet}$ is $(n+r-1)$-skeletal by 33.18 (i), from the description above we see that we only need $P^{r-1} \mathfrak{Z}\left\{\Omega^{k} \overline{\mathbf{W}}^{N}\right\}$ in order to calculate $d_{r}$, and thus $E_{r+1}^{* *}$. Finally, by $2.24 \quad P^{r-1} \mathfrak{Z}$ is completely determined by the $(r-1)$-truncated $R$-mapping algebra $P^{r-1} \mathfrak{W}_{\bullet}$, and this in turn depends only on $P^{r-1} \mathfrak{M}^{\mathrm{St}, R} \mathbf{Y}$, up to $E_{2}$-equivalence.

4.15. Corollary. For any $\mathbf{Z} \in \mathcal{S}_{*}$ and $R=\mathbb{F}_{p}$ or $\mathbb{Q}$, the mapping space functor $\operatorname{map}_{*}(\mathbf{Z},-)$ is a level homotopy functor $\mathcal{S}_{R} \rightarrow \mathcal{S}_{*}$.

\section{REFERENCES}

[A] J.F. Adams, "On the structure and applications of the Steenrod algebra", Comm. Math. Helv. 32 (1958), pp. 180-214.

[BBD] B. Badzioch, D. Blanc, \& W. Dorabiała, "Recognizing mapping spaces", J. Pure $E$ Appl. Alg. 218 (2013), pp. 181-196.

[Bau] H.-J. Baues, The algebra of secondary cohomology operations, Birkhäuser Prog. in Math. 247, Basel, 2006.

[BB1] H.-J. Baues \& D. Blanc, "Stems and Spectral Sequences", Alg. \& Geom. Top., 10 (2010), pp. 2061-2078.

[BB2] H.-J. Baues \& D. Blanc, "Comparing cohomology obstructions", J. Pure Appl. Alg., 215 (2011), pp. 1420-1439.

[BB3] H.-J. Baues \& D. Blanc, "Higher order derived functors and the Adams spectral sequence", J. Pure Appl. Alg., 219 (2015), pp. 199-239.

[BJ2] H.-J. Baues \& M.A. Jibladze, "Dualization of the Hopf algebra of secondary cohomology operations and the Adams spectral sequence", J. K-Theory 7 (2011), pp. 203-347.

[BBS] S. Basu, D. Blanc, \& D. Sen, "Higher structure in the unstable Adams spectral sequence", preprint, 2018.

[BCM] M. Bendersky, E.B. Curtis, \& H.R. Miller, "The unstable Adams spectral sequence for generalized homology", Topology 17 (1978), pp. 229-248.

[Bi] A. Bittner, "Spaces With Complexity One", preprint, 2017 , arXiv:1712.04009

[Bl1] D Blanc, "A Hurewicz spectral sequence for homology", Trans. AMS 318 (1990), pp. 335354

[B12] D Blanc, "Mapping spaces and $M-C W$ complexes", Forum M. 9 (1997), pp. 367-382.

[Bl3] D. Blanc, "Realizing coalgebras over the Steenrod algebra", Topology 40 (2001), pp. 9931016.

[BDG] D. Blanc, W.G. Dywer, \& P.G. Goerss, "The realization space of a $\Pi$-algebra: a moduli problem in algebraic topology", Topology 43 (2004), pp. 857-892. 
[BJT1] D. Blanc, M.W. Johnson, \& J.M. Turner, "On realizing diagrams of П-algebras", Alg. \& Geom. Top. 6 (2006), pp. 763-807.

[BJT2] D. Blanc, M.W. Johnson, \& J.M. Turner, "Higher homotopy invariants for spaces and maps", preprint, 2019.

[BS1] D. Blanc \& D. Sen, "Higher cohomology operations and R-completion", Alg. \& Geom. Top. 18 (2018), pp. 247-312.

[BS2] D. Blanc \& D. Sen, "Mapping spaces and R-completion", J. Homotopy \& Related Structures 13 (2018), pp. 635-671.

[Bor] F. Borceux, Handbook of Categorical Algebra, Vol. 2: Categories and Structures, Encyc. Math. \& its Appl., 51, Cambridge U. Press, Cambridge, UK, 1994.

[Bou1] A.K. Bousfield, "Homotopy Spectral Sequences and Obstructions", Israel J. Math., 66 (1989), pp. 54-104.

[Bou2] A.K. Bousfield, "Cosimplicial resolutions and homotopy spectral sequences in model categories", Geom. ES Topology 7 (2003), pp. 1001-1053.

[Bou3] A.K. Bousfield, "On the telescopic homotopy theory of spaces", Trans. AMS 353 (2001), pp. 2391-2426.

[BC] A.K. Bousfield \& E.B. Curtis, "A spectral sequence for the homotopy of nice spaces", Trans. AMS 151 (1970), 457-478.

[BF] A.K. Bousfield \& E.M. Friedlander, "Homotopy theory of $\Gamma$-spaces, spectra, and bisimplicial sets", in M.G. Barratt \& M.E. Mahowald, eds., Geometric Applications of Homotopy Theory, II Springer-Verlag Lec. Notes Math. 658, Berlin-New York, 1978, 80-130

[BK1] A.K. Bousfield \& D.M. Kan, "The homotopy spectral sequence of a space with coefficients in a ring", Topology 11 (1972), pp. 79-106.

[BK2] A.K. Bousfield \& D.M. Kan, Homotopy Limits, Completions, and Localizations, Springer Lec. Notes Math. 304, Berlin-New York, 1972.

[BK3] A.K. Bousfield \& D.M. Kan, "A second quadrant homotopy spectral sequence", Trans. AMS 177 (1973), pp. 305-318.

[CDI] W. Chachólski, W.G. Dwyer, \& M. Intermont, "The A-complication of spaces", J. London Math. Soc. 65 (2002), 204-222.

[CF] J.D. Christensen \& M. Frankland, Higher Toda brackets and the Adams spectral sequence in triangulated categories", Alg. $\mathscr{G}$ Geom. Top. 17 (2017), pp. 2687-2735.

[DK1] W.G. Dwyer \& D.M. Kan, "Singular functors and realization functors", Proc. Kon. Ned. Akad. Wet. 2 (1984), pp. 147-153,

[DK2] W.G. Dwyer \& D.M. Kan, "The enveloping ring of a П-algebra", in S.M. Salamon, B. Steer, \& W.A. Sutherland, eds., Advances in Homotopy Theory (Cortona, 1988), Lond. Math. Soc. Lec. Notes Ser. 139, Cambridge U. Press, Cambridge, UK, 1989, pp. 49-60.

[DKSS] W.G. Dwyer \& D.M. Kan, J.F. Smith, \& C.R Stover, "A П-algebra spectral sequence for function spaces", Proc. AMS 120 (1994), pp. 615-621.

[DKS1] W.G. Dwyer \& D.M. Kan, \& C.R Stover, "An $E^{2}$ model category structure for pointed simplicial spaces", J. Pure Appl. Alg. 90 (1993), pp. 137-152.

[DKSt2] W.G. Dwyer, D.M. Kan, \& C.R. Stover, "The bigraded homotopy groups $\pi_{i, j} X$ of a pointed simplicial space", J. Pure Appl. Alg. 103 (1995), pp. 167-188.

[EM] S. Eilenberg \& J.C. Moore", Foundations of relative homological algebra Memoirs of the AMS 55, AMS, Providence, RI, 1965.

[EKMM] A.D. Elmendorf, I. Křriž, M.A. Mandell, \& J.P. May, Rings, modules, and algebras in stable homotopy theory, AMS, Providence, RI, 1997.

[GJ] P.G. Goerss \& J.F. Jardine, Simplicial Homotopy Theory, Progress in Mathematics 179, Birkhäuser, Basel-Boston, 1999.

[H] P.S. Hirschhorn, Model Categories and their Localizations, Math. Surveys \& Monographs 99, AMS, Providence, RI, 2002.

[HSS] M.A. Hovey, B.E. Shipley, J.H. Smith, "Symmetric spectra", Jour. AMS 13 (2000), 149208

[I] L. Illusie, Complexe cotangent et déformations. II, Lec. Notes Math. 283, Springer, BerlinNew York, 1972. 
[J] J.F. Jardine, "Bousfield's $E_{2}$ Model Theory for Simplicial Objects", in P.G. Goerss \& S.B. Priddy, eds., Homotopy Theory: Relations with Algebraic Geometry, Group Cohomology, and Algebraic K-Theory, AMS, Providence, RI, 2004, pp. 305-319

[K] G.M. Kelly, Basic concepts of enriched category theory, Cambridge U. Press, Cambridge, UK, 1982.

[L] M.G. Lydakis, "Smash products and Г-spaces", Math. Proc. Camb. Phil. Soc. 126 (1999), pp. 311-328.

[Mc] S. Mac Lane, Categories for the Working Mathematician, Springer-Verlag, Berlin-New York, 1971.

[Ma1] J.P. May, The Geometry of Iterated Loop Spaces, Springer Lec. Notes Math. 271, BerlinNew York, 1972.

[Ma2] J.P. May, Simplicial Objects in Algebraic Topology, U. Chicago Press, Chicago-London, 1967.

[Mo] J.C. Moore, "Semi-simplicial complexes and Postnikov systems", in J. Adem et al., eds., Symposium internacional de topología algebraica, U. Nac. Aut. Mexico \& UNESCO, Mexico City, 1958, pp. 232-247i

[N] S.P. Novikov, "Operation rings and spectral sequences of the Adams type in extraordinary cohomology theories", Doklady Ak. Nauk SSSR 172 (1967), pp. 33-36.

[Q1] D.G. Quillen, "Spectral sequences of a double semi-simplicial group", Topology 5 (1966), pp. $155-156$.

[Q2] D.G Quillen, Homotopical Algebra, Springer, Berlin-New York, 1967.

[R] D.C. Ravenel, Complex Cobordism and Stable Homotopy Groups of Spheres, Academic Press, Orlando, 1986.

[Sc] L. Schwartz, Unstable Modules over the Steenrod Algebra and Sullivan's Fixed Point Set Conjecture, U. Chicago Press, Chicago-London, 1994.

[Sta] J.D. Stasheff, H-spaces from a Homotopy Point of View, Springer Lec. Notes Math. 161, Berlin-New York, 1970.

[Sto] C.R. Stover, "A Van Kampen spectral sequence for higher homotopy groups", Topology 29 (1990), pp. 9-26.

[W] C.A. Weibel, An Introduction to Homological Algebra, Cambridge U. Press, Cambridge, UK, 1994

Max-Planck-Institut für Mathematik, Vivatsgasse 7, 53111 Bonn, Germany

E-mail address: baues@mpim-bonn.mpg.de

Department of Mathematics, University of Haifa, 3498838 Haifa, Israel

E-mail address: blanc@math.haifa.ac.il

Department of Mathematics, University of Haifa at Oranim, 3600600 Tivon, Israel

E-mail address: chorny@math.haifa.ac.il 